\title{
HIDDEN MATRICES
}

\section{S. LEVY, J. C. ROBSON, and J. T. STAFFORD}

[Received 24 August 1992-Revised 1 July 1993]

\begin{abstract}
We investigate subrings of an $n \times n$ matrix ring which, despite appearing otherwise, are themselves full rings of $n \times n$ matrices; that is, are hidden matrices. In general, this problem is subtle, but we give fairly complete results in a number of situations. For example, we prove:

THEOREM A. Let $K$ be an ideal of a ring $R$ and suppose that $T=\left(R_{i j}\right)$ is a tiled subring of $M_{n}(R)$ containing $M_{n}(K)$. Suppose that $R_{i i}=R_{i j}$ for all $i$ and $j$ and that $R_{i i} / K \cong M_{n}(D)$, for some ring $D$. Then $T \cong M_{n}(S)$, for a ring $S$ that we describe explicitly.

The subtleties are illustrated by the following theorem:

THEOREM B. Let $\mathbb{H}$ denote the ring of integer quaternions and let $p$ be an odd prime number. Set $R=\mathbb{H}+M_{2}(p \llbracket)$, where $\mathbb{H}$ is identified with the ring of scalar matrices inside $M_{2}(\mathbb{H})$. Then $R \cong M_{2}(S)$, for some ring $S$, if and only if $p \equiv 1(\bmod 4)$.
\end{abstract}

\section{Introduction}

The general theme of this paper is summed up by:

1.1. Question. Let $K$ be a non-zero ideal of a ring $R$. Suppose that $T$ is a ring such that $M_{n}(K) \subset T \subseteq M_{n}(R)$ and $\bar{T}=T / M_{n}(K) \cong M_{n}(D)$ for some ring $D$. What extra conditions will ensure that $T \cong M_{n}(S)$, for some ring $S$ ?

This question is an abstraction of a very specific question posed by Chatters in the introduction to [2]. Write $\mathbb{H}=\mathbb{Z}[i, j, k]$ for the ring of integer quaternions and let $\mathbb{H}_{p}$ denote the localization of $\mathbb{H}$ at some odd prime number $p$. Consider the following tiled subrings of $M_{2}(\mathbb{H})$ and $M_{2}\left(\mathbb{H}_{p}\right)$, respectively:

$$
T=\left(\begin{array}{cc}
\mathbb{H} & p \mathbb{H} \\
\mathbb{H} & \mathbb{H}
\end{array}\right), \quad T^{\prime}=\left(\begin{array}{cc}
\mathbb{H}_{p} & p \mathbb{H}_{p} \\
\mathbb{H}_{p} & \mathbb{H}_{p}
\end{array}\right) .
$$

Chatters showed that $T^{\prime}$ is isomorphic to a $2 \times 2$ matrix ring (over a suitable ring) and asked:

\subsection{Question. Is the ring $T$ defined in (1.1.1) a full $2 \times 2$ matrix ring?}

This question does now have a positive answer, thus making $T$ into a 'hidden matrix ring'. This has been proved, independently, by Chatters [3] and Robson [17] but both use calculations too specific to the integer quaternions to be of value in tackling Question 1.1. However, observe that the following theorem, which is 
one of the main consequences of this paper, also answers Question 1.2, since the ideal $K=p \mathbb{H}$ satisfies $\mathbb{H} / K \cong M_{2}(\mathbb{Z} /(p))$.

1.3. Theorem. (See Theorem 4.6.) Let $K$ be an ideal of a ring $R$ and suppose that $T=\left(R_{i j}\right)$ is a tiled subring of $M_{n}(R)$ containing $M_{n}(K)$. Suppose that $R_{i i}=R_{i j}$ for all $i$ and $j$ and that $R_{i i} / K \cong M_{n}(D)$, for some ring $D$. Then $T \cong M_{n}(S)$, for some ring $S$.

The idea behind Theorem 1.3, and the relationship between Questions 1.1 and 1.2 , are best explained by considering the ring $T$ from (1.1.1) in greater detail. Set $\overline{\mathbb{Z}}=\mathbb{Z} /(p)$. Then

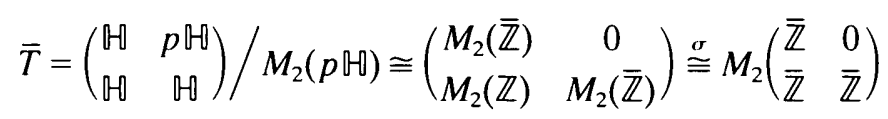

is a $2 \times 2$ matrix ring. (Thus, $T$ does satisfy the hypotheses of Question 1.1.) The critical observation is that the final isomorphism $\sigma$ in this equation can be accomplished by conjugation by the $4 \times 4$ permutation matrix $(2,3)$, thought of as an element of $M_{4}(\overline{\mathbb{Z}})$. Consequently, the key step in answering Question 1.2 lies in proving that some isomorphism of the form (1.3.1) lifts to an inner automorphism $\tau$ of the $2 \times 2$ matrix ring $M_{2}(\mathbb{H})$ as it is then easy to prove that $\tau(T)$ is a $2 \times 2$ matrix ring. Similar lifting questions lie at the heart of the proof of Theorem 1.3.

Such lifting questions are considered in $\S 3$. The main result of that section, Theorem 3.3, concerns projective right modules $P_{1}, \ldots, P_{r}$ over a ring $A$ such that each $P_{i}$ has a factor module $\bar{P}_{i} \cong U^{(n(i))}$, for some fixed module $U$. Theorem 3.3 states, roughly, that any automorphism of $\bar{P}_{1} \oplus \ldots \oplus \bar{P}_{r}$ which merely permutes the copies of $U$ can be lifted to an automorphism of $P_{1} \oplus \ldots \oplus P_{r}$. This is then used in $\S 4$ to prove various Hidden Matrix Theorems. Given a matrix ring $E=M_{s}(D)$, identify $M_{t}(E)$ with $M_{t s}(D)$. A subring $\bar{T}$ of $M_{t s}(D)$ is called permutationisomorphic to a $t \times t$ matrix ring if there exists a $t s \times t s$ permutation matrix $\alpha$ such that $\alpha^{-1} \bar{T} \alpha=M_{t}(C)$ for some subring $C$ of $E=M_{s}(D)$. This formalizes the comments made after (1.3.1). One of our more definitive answers to Question 1.1 is the following generalization of Theorem 1.3:

1.4. Theorem. (See Theorem 4.2.) Let $K$ be an ideal of a ring $R$ such that $R / K \cong M_{n}(D)$, for some ring $D$, and let $T$ be a ring such that $M_{n}(K) \subset T \subseteq M_{n}(R)$. Suppose that the subring $\bar{T}=T / M_{n}(K)$ of $M_{n}(R) / M_{n}(K)=M_{n^{2}}(D)$ is permutation-isomorphic to an $n \times n$ matrix ring. Assume that either

(i) $T / M_{n}(K)$ is a tiled subring of $M_{n^{2}}(D)$; or

(ii) the centre $Z(R)$ of $R$ contains an $n$th root of -1 .

Then $T \cong M_{n}(S)$, for some ring $S$.

We remark that the theorem becomes false if one replaces 'permutationisomorphic' by 'isomorphic' (see Corollary 5.16). Consequently, one cannot generalize Theorem 1.3 by replacing the conditions ' $R_{i i}=R_{j j}$ for all $i$ and $j$ and $R_{i i} / K \cong M_{n}(D)$ for some ring $D$ ' by the simpler hypothesis 'for each $i, R_{i i} / K$ is isomorphic to an $n \times n$ matrix ring'.

Note that condition (ii) of Theorem 1.4 is always satisfied when $n$ is odd, the 
needed root being -1 . For even-sized matrices the situation is considerably more subtle. As a deceptively simple illustration, suppose that $n$ is any positive integer and let $K$ be an ideal of a ring $R$ such that $R / K \cong M_{n}(D)$. Identify $R$ with the ring of scalar matrices in $M_{n}(R)$ and set $T_{n}=R+M_{n}(K)$. Clearly, $T_{n} / M_{n}(K) \cong R / K \cong M_{n}(D)$ and it is easy to show that this is a permutationisomorphism. Thus, if $n$ is odd, or if $Z(R)$ contains an $n$th root of -1 , then Theorem 1.4(ii) implies that $T_{n} \cong M_{n}(S)$ for some ring $S$. More generally:

1.5. Theorem. (See Theorem 4.8.) Let $K$ be an ideal of a ring $R$ such that $R / K \cong M_{n}(D)$, for some ring $D$. Set $T_{n}=R+M_{n}(K)$.

(i) If either $n \equiv 0,1,3(\bmod 4)$ or $Z(R)$ contains a square root of -1 , then $T_{n} \cong M_{n}(S)$ for some ring $S$.

(ii) For all $n, M_{n}\left(T_{n}\right)$ is a full $n^{2} \times n^{2}$ matrix ring.

When $n \equiv 2(\bmod 4)$ and $\sqrt{ }-1 \notin Z(R)$ there is no such simple answer. For example:

1.6. Theorem. (See Proposition 6.8.) Let $p$ be an odd prime number and $T_{2}=\mathbb{H}+M_{2}(p H)$. Then $T_{2} \cong M_{2}(S)$, for some ring $S$, if and only if $p \equiv 1(\bmod 4)$.

As is proved in Proposition 6.10, similar examples exist for any $n \equiv 2(\bmod 4)$. These results are proved by means of a careful analysis of the structure of rings of the form $T_{2}=R+M_{2}(K)$ (see Theorem 6.5 in particular). Note that these examples also show that one cannot delete the hypotheses (i) and (ii) from Theorem 1.4. Moreover, as $i \in \mathbb{H}$, one also cannot replace the assumption of part (ii) of Theorem 1.4 by the hypothesis that there exists $x \in R$ such that $x^{n}=-1$.

The reason why these subtleties should exist stems from an issue that has been suppressed up to now. Namely, the 'permutation' matrices that we need to consider in this paper are a little more general than the usual ones, in that their non-zero entries (one in each row and column) are allowed to be \pm 1 . We call these signed permutation matrices. Let $\phi: R \rightarrow M_{n}(D)$ be a surjective ring homomorphism and $s \geqslant 2$ an integer. Then Corollary 3.4 implies, in particular, that every signed permutation matrix in $M_{n s}(D)$ of determinant 1 can be lifted to a unit in $M_{s}(R)$. The significance of this fact is as follows. Let $\alpha$ be the $n^{2} \times n^{2}$ permutation matrix given by the hypotheses of Theorem 1.4. If $n$ is odd, then one can always replace $\alpha$ by a signed permutation of determinant +1 and so Corollary 3.4 implies that it can be lifted. If $n$ is even, conditions (i) and (ii) of Theorem 1.4 amount to sufficient conditions for such a replacement to exist.

Curiously, the ideas behind Corollary 3.4 also yield a new, strengthened form of Schanuel's Lemma (see Lemma 3.7) which, in turn, has some unexpected consequences. For example, suppose that $A$ and $B$ are maximal right ideals of a ring $R$ such that $R / A \cong R / B$. Then $A \oplus A \cong A \oplus B$. Consequently, given any four maximal right ideals $A_{i}$ of $R$, with $R / A_{i} \cong R / A_{j}$ for all $i$ and $j$, then $A_{1} \oplus A_{2} \cong A_{3} \oplus A_{4}$ (see Corollary 3.8 ).

In the one section we have yet to discuss, $\S 5$, we view Question 1.1 from a different perspective, in that we drop the assumption that the appropriate isomorphism be a permutation isomorphism but we impose the additional 
conditions that both $R$ and $T$ are right Noetherian and that both $\bar{R}=R / K$ and $\bar{T}$ are semilocal. Surprisingly, these assumptions imply that $T$ is a tiled subring of $M_{n}(\tilde{R})$, for some ring $\tilde{R}$ with the property that $M_{n}(R) \cong M_{n}(\tilde{R})$ (see Theorem 5.8). With somewhat stronger conditions one finds that $T$ is very close to being a full matrix ring:

1.7. Theorem. (See Theorems 5.6 and 5.18.) Let $K, R, T$ be as in Question 1.1 and assume that $R$ and $T$ are prime, right Noetherian rings of Krull dimension 1. Then there exist a ring $S$ Morita equivalent to $T$ and a projective right ideal $L$ of $S$ such that $T \cong \operatorname{End}_{S}\left(S^{(n-1)} \oplus L\right)$.

The ring $T$ of Theorem 1.7 need not be an $n \times n$ matrix ring; the example alluded to after Theorem 1.4 is constructed in just this manner. Note that Theorem 1.7 echoes a standard structure theorem [15, Corollary 5.7.9] for classical orders. Appropriately, a key tool in this section is a generalization of the classical notion of the genus of a projective module.

In conclusion, this paper demonstrates the ubiquity of hidden matrix rings. To emphasise this, we mention a result that provides a plethora of examples. Let $R$ be any non-commutative domain that, like the integer quaternions, arises in integral representation theory and let the dimension of the quotient division ring of $R$ over its centre be $n^{2}$. Build a random $n \times n$ tiled matrix ring $T$, using $R$ for diagonal tiles and non-zero ideals of $R$ for off-diagonal tiles. Then Theorem 5.17 gives a sense in which $T$ is almost always isomorphic to a full $n \times n$ matrix ring.

Acknowledgements. The research of Levy and Stafford was supported in part by NSF grants and that of Robson by the EEC. Levy was a visiting professor at Wayne State University during the year that this research was begun. $\mathrm{He}$ is grateful for their hospitality and support.

The authors are also grateful to D. S. Passman for pointing out an error in the original version of $\S 6$.

\section{Preliminary results}

Throughout this paper, all rings contain an identity element and this is assumed to be inherited by any subring and by any homomorphic image. Similarly, all modules are unital. The following notation will be used throughout the paper. A set of $n \times n$ matrix units in a ring $M$ is a set of $n^{2}$ elements $e_{i j} \in M$ $(i, j \in\{1,2, \ldots, n\})$ such that $e_{i j} e_{j k}=e_{i k}, e_{i j} e_{h k}=0$ if $j \neq h$, and $\sum_{i} e_{i i}=1$. If $M=M_{n}(R)$ for some ring $R$, and $e_{i j}$ is the matrix with 1 in position $(i, j)$ and zeros elsewhere, then $\left\{e_{i j}\right\}$ is a set of $n \times n$ matrix units, the standard matrix units of $M_{n}(R)$. By a tiled subring $T=\left(R_{i j}\right)$ of $M_{n}(R)$ we mean a subring of the form $T=\bigoplus_{i j} R_{i j} e_{i j}$ where each $R_{i j}$ is an additive subgroup of $R$. If $P$ is a module over a ring $R$, then the direct sum of $n$ copies of $P$ will be denoted by $P^{(n)}$, with the convention that $P^{(0)}=0$.

The following well-known result is easily proved and will be used frequently in the paper. 
2.1. Lemma. Let $M$ be a ring.

(i) Then $M \cong M_{n}(R)$, for some ring $R$ if and only if $M$ contains a set of $n \times n$ matrix units.

(ii) (Therefore) if $M$ is an $n \times n$ matrix ring, so is every ring containing $M$, and every homomorphic image of $M$.

(iii) Suppose that $M \cong \operatorname{End}_{S}(Q)$, for some right module $Q$ over a ring $S$. Then, $M \cong M_{n}(R)$, for some ring $R$, if and only if there exists a right $S$-module $X$ such that $Q \cong X^{(n)}$, as right $S$-modules.

Throughout the paper, $\mathbb{H}=\mathbb{Z}[i, j, k]$ will denote the ring of integer quaternions and we will write $\mathbf{H}=\mathbb{Z}\left[i, j, k, \frac{1}{2}(1+i+j+k)\right]$ for the (unique) maximal $\mathbb{Z}$-order, in the quotient division ring of $\mathbb{H}$, that contains $\mathbb{H}$. The following well-known property of these algebras, proved, for example, in [3, Appendix], will be used in many places in this paper.

2.2. Lemma. For every odd prime number $p$, the rings $\mathbb{H} / p \mathbb{H}$ and $\mathbf{H} / p \mathbf{H}$ are isomorphic to $M_{2}(\mathbb{Z} / p \mathbb{Z})$.

\section{Lifting permutations}

Let $P_{1}, \ldots, P_{r}$ be projective modules over a ring $R$ such that each $P_{i}$ has a factor module $\bar{P}_{i}$ which is a direct sum of copies of a fixed module $U$. The main result of this section, Theorem 3.3, shows how to lift certain permutations (or signed permutations) of the copies of $U$ to automorphisms of $P_{1} \oplus \ldots \oplus P_{r}$. This is used in $\S 4$ to uncover various hidden matrix rings. Perhaps surprisingly, Theorem 3.3 can be viewed as an extension of Schanuel's Lemma. The final portion of this section makes the formal connection with Schanuel's Lemma, as it has some interesting, albeit easy consequences. In particular, this provides a hidden matrix theorem that is not covered by the more general results of $\S 4$.

For some $r \geqslant 2$, let $M_{1}, \ldots, M_{r}$ be right modules over a ring $R$ and write $M=M_{1} \oplus \ldots \oplus M_{r}$. Given $\sigma \in \operatorname{Hom}\left(M_{j}, M_{i}\right)$ with $i \neq j$, let $\sigma e_{i j}$ denote the endomorphism of $M$ which maps $M_{j}$ to $M_{i}$ via $\sigma$ and is zero on $M_{k}$, for $k \neq j$. A transvection is any endomorphism of $M=M_{1} \oplus \ldots \oplus M_{r}$ of the form $E_{i j}(\sigma)=$ $1+\sigma e_{i j}$, where $i \neq j$ and $\sigma \in \operatorname{Hom}_{R}\left(M_{j}, M_{i}\right)$. This is an automorphism of $M$ with inverse $E_{i j}(-\sigma)$. The elementary group $E\left(M_{1}, \ldots, M_{r}\right)$ is the subgroup of Aut $M$ generated by all transvections of $M_{1} \oplus \ldots \oplus M_{r}$. If $M_{i} \cong M_{1}$ for all $i$, we will write $E\left(r, M_{1}\right)$ for this elementary group. Note that this may be identified with $E(r, S)$, the usual elementary group of $r \times r$ matrices over $S=$ End $M_{1}$. The starting point for this section is the observation that elementary transformations can be lifted.

3.1. Lemma. Let $R$ be $a$ ring and $r \geqslant 2$ an integer. Let $P=P_{1} \oplus \ldots \oplus P_{r}$ be a projective right $R$-module and let $\vec{P}_{i}$ be a factor module of $P_{i}$. Then each $\bar{\alpha} \in E\left(\bar{P}_{1}, \ldots, \bar{P}_{r}\right)$ can be lifted to an automorphism $\alpha \in E\left(P_{1}, \ldots, P_{r}\right)$.

Proof. Use the proof of [1, Proposition IV.3.3].

Keep the notation of Lemma 3.1. The basic idea behind the results in this section is that many automorphisms of $\bar{P}$, in particular those that just permute 
direct summands of the $\bar{P}_{i}$, can more or less be written as products of transvections and hence be lifted to automorphisms of $P$. To make this precise, we need to introduce some more terminology. Let $U$ be a right $R$-module and $n \geqslant 2$ be an integer. Given $i \neq j$, let $\langle i, j\rangle$ denote the automorphism of $U^{(n)}$ that multiplies the $i$ th entry by -1 and then interchanges it with the $j$ th entry, leaving all other entries unaffected. We call $\langle i, j\rangle$ a signed transposition. More generally, a signed permutation is any automorphism of $U^{(n)}$ that permutes the copies of $U$, multiplying some of them by -1 . This can be viewed as an $n \times n$ matrix, acting on the columns of $U^{(n)}$ by left multiplication, in which case it is simply an $n \times n$ matrix with exactly one non-zero entry, \pm 1 , in each row and column. The group of all signed permutations of $U^{(n)}$ will be denoted by $S_{n}^{ \pm}(U)$. This contains, as subgroups, the usual permutation group $S_{n}(U)$ and the group $S_{n}^{+}(U)$ of all signed permutations of determinant 1. Finally, let $\Delta_{n}(U)$ denote the subgroup of $S_{n}^{ \pm}(U)$ which comprises all the diagonal matrices with entries \pm 1 and let $\delta_{i j}$ denote the diagonal matrix whose entries along the diagonal are -1 in the $i$ th and $j$ th positions and +1 elsewhere.

The next lemma, while elementary, demonstrates the advantage of working with signed permutations and, more generally, with $S_{n}^{+}(U)$. For, it shows that any $\alpha \in S_{n}^{+}(U)$ actually lies in $E(n, U)$ and so, by Lemma 3.1 , can be lifted. In many of the applications, we will be concerned with lifting automorphisms of factor modules to automorphism of the original module, in which case the multiplication of various summands by -1 is basically irrelevant.

3.2. Lemma. Let $U$ be any module over some ring $R$ and fix distinct integers $i, j$, $k$ with $1 \leqslant i, j, k \leqslant n$. Then the following identities hold in $\operatorname{End}_{R}\left(U^{(n)}\right)$ :

(i) $\langle i, j\rangle=E_{i j}(1) E_{j i}(-1) E_{i j}(1)=\langle j, i\rangle^{-1}=\langle j, i\rangle^{3}$;

(ii) $\langle i, j\rangle^{2}=\delta_{i j}$;

(iii) $E_{i j}(\sigma)=\langle j, k\rangle E_{i k}(\sigma)\langle k, j\rangle$, for any $\sigma \in \operatorname{End}(U)$;

(iv) $S_{n}^{+}(U)$ is generated by the signed transpositions $\{\langle i, j\rangle: 1 \leqslant i<j \leqslant n\}$;

(v) $S_{n}^{+}(U) \subseteq E(n, U)$.

Proof. (i) (ii) (iii) These are readily checked.

(iv) Clearly $S_{n}^{ \pm}(U)$ is generated by $\Delta_{n}(U)$, together with the $\langle i, j\rangle$. Moreover, $\langle i, j\rangle \in S_{n}^{+}(U)$, for $i \neq j$. If $\delta \in \Delta_{n}(U)$ satisfies det $\delta=1$ then $\delta$ has an even number of negative entries and so, by part (ii), is a product of signed transpositions, as required.

(v) This follows from parts (iv) and (i).

We can now give the main result of this section.

3.3. Theorem (Lifting signed permutations). For $r \geqslant 2$, let $P_{1}, \ldots, P_{r}$ be projective right modules over a ring $R$ and set $P=P_{1} \oplus \ldots \oplus P_{r}$. Suppose that there are a right $R$-module $U$ and integers $n(i) \geqslant 1$ such that, for each $i$, there is $a$ surjection $\phi_{i}: P_{i} \rightarrow \bar{P}_{i}=U^{n(i)}$. Write $\phi=\left(\phi_{1}, \ldots, \phi_{r}\right)$ for the induced surjection $\phi: P \rightarrow U^{(n)}$, where $n=\sum n(i)$.

(i) Each $\bar{\alpha} \in E(n, U)$, and hence each $\bar{\alpha} \in S_{n}^{+}(U)$, can be lifted to an automorphism of $P$.

(ii) If there exists $\bar{\beta} \in S_{n}^{ \pm}(U)$ with $\operatorname{det} \bar{\beta}=-1$ which lifts to $\beta \in$ Aut $P$, then each $\bar{\alpha} \in S_{n}^{ \pm}(U)$ can be lifted to an automorphism $\alpha$ of $P$. 
(iii) Suppose that, for some $i$, the centre $Z(R)$ of $R$ contains an $n(i)$ th root of -1 . Then each $\bar{\alpha} \in S_{n}^{ \pm}(U)$ lifts to an $\alpha \in$ Aut $P$.

Remarks. In each part of the theorem we actually show that $\alpha \in E\left(P_{1}, \ldots, P_{r}\right)$. Part (iii) of the theorem implies, in particular, that every element of $S_{n}^{ \pm}(U)$ can be lifted if some $n(i)$ is odd.

Proof. (i) In order to identify the different copies of $U$, write $U^{(n)}=$ $\bigoplus \bar{P}_{i}=U_{1} \oplus \ldots \oplus U_{n}$, where $\bar{P}_{i}=U_{v(i)+1} \oplus \ldots \oplus U_{v(i)+n(i)}$ for $v(i)=\sum_{j=1}^{i-1} n(j)$.

By Lemma 3.2(v), $S_{n}^{+}(U) \subseteq E(n, U)$ and so it suffices to prove part (i) when $\bar{\alpha}$ is a transvection. Moreover, by reordering the $U_{i}$ and $P_{j}$, we may assume that $\bar{\alpha}=1+\sigma e_{1}$, for some $\sigma \in \operatorname{End}(U)$ and $t \geqslant 2$. If $t \geqslant n(1)+1$, then $U_{t}$ is a summand of $\bar{P}_{j}$ for some $j \geqslant 2$ and hence $\bar{\alpha} \in E\left(\bar{P}_{1}, \ldots, \bar{P}_{r}\right)$. Thus, $\bar{\alpha}$ can be lifted by Lemma 3.1. Alternatively, suppose that $t \leqslant n(1)$ and set $s=n(1)+1$; thus $U_{s}$ is a summand of $\bar{P}_{2}$. Lemma 3.2 (iii) implies that there is a factorization $\bar{\alpha}=$ $E_{1 t}(\sigma)=\langle t, s\rangle E_{1 s}(\sigma)\langle s, t\rangle$ in $E(n, U)$. Thus, by the choice of $s$ and Lemma 3.2(i), $\bar{\alpha} \in E\left(\bar{P}_{1}, \ldots, \bar{P}_{r}\right)$. Therefore, by Lemma 3.1 again, $\bar{\alpha}$ can be lifted.

(ii) If $\operatorname{det} \bar{\alpha}=1$ then part (i) applies. Otherwise $\operatorname{det} \bar{\beta} \bar{\alpha}=1$ and so $\bar{\beta} \bar{\alpha}$ can be lifted to, say, $\gamma \in \operatorname{Aut}_{R}(P)$. Thus $\bar{\alpha}$ lifts to $\alpha=\beta^{-1} \gamma$.

(iii) By reordering the $P_{j}$, we may assume that $i=1$. Set $s=n(1)$ and let $\xi$ be an $s$ th root of -1 in $Z(R)$. Write $\gamma$ for the automorphism of $P$ that multiplies $P_{1}$ by $\xi$ and is the identity on $P_{j}$ for $j>1$. Then $\gamma$ maps $\operatorname{ker} \phi$ onto itself and therefore induces an automorphism $\bar{\gamma}$ of $U^{(n)}$. The action of $\bar{\gamma}$ is easy to describe: it equals left multiplication by a diagonal $n \times n$ matrix $\bar{\gamma}=\operatorname{diag}(\xi, \ldots, \xi, 1, \ldots, 1)$, where $\xi$ appears $s$ times.

Let $u, v$ be units in a ring $A$. Then the Whitehead Lemma [1, Proposition V.1.7], implies that $\left(\begin{array}{ll}u & 0 \\ 0 & v\end{array}\right) \varepsilon=\left(\begin{array}{cc}u v & 0 \\ 0 & 1\end{array}\right)$ for some $\varepsilon \in E(2, A)$. Therefore, by induction, there exists $\delta \in E(n, U)$ such that

$$
\bar{\beta}=\bar{\gamma} \delta=\operatorname{diag}\left(\xi^{s}, 1,1, \ldots, 1\right)=\operatorname{diag}(-1,1,1, \ldots, 1) .
$$

By part (i) and the last paragraph, $\delta$ and $\bar{\gamma}$ can be lifted to automorphisms of $P$. Thus $\bar{\beta}$ can be lifted and so the result follows from part (ii).

The preceding theorem will be applied in the following form in $\S 4$ to prove various results about hidden matrices.

3.4. Corollary. Let $\phi: R \rightarrow M_{s}(D)$ be a surjective ring homomorphism, for some $s \geqslant 2$. Given $r \geqslant 2$, let $\phi_{r}: M_{r}(R) \rightarrow M_{r s}(D)$ be the induced homomorphism obtained by letting $\phi$ act on each entry.

(i) Each matrix $\bar{\alpha} \in E(r s, D)$ can be lifted via $\phi_{r}$ to a unit $\alpha \in E(r, R) \subset M_{r}(R)$.

(ii) Hence, each signed permutation matrix $\bar{\alpha} \in S_{r s}^{+}(D)$ can be so lifted.

(iii) If $Z(R)$ contains an sth root of -1 , then each $\bar{\alpha} \in S_{r s}^{ \pm}(D)$ can be lifted.

Proof. Note that $M_{s}(D)=\bigoplus_{i=1}^{s} e_{i i} M_{s}(D)$ and, for $1 \leqslant i \leqslant s$, one has $e_{i i} M_{s}(D) \cong e_{11} M_{s}(D)=U$, say. Now apply Theorem 3.3 in the case where $P_{i}=R$ and $\bar{P}_{i}=M_{s}(D) \cong U^{(s)}$ for each $i$. 
Perhaps the following special case of part (i) of the previous corollary deserves mention (take $R=M_{s}(D)$ and $\phi$ the identity map).

3.5. Corollary. For any ring $D$ and integers $r$, $s$ with $r \geqslant 2$,

$$
E\left(r, M_{s}(D)\right)=E(r s, D) \text {. }
$$

3.6. ExAmple. As a simple application of Corollary 3.4, the reader is invited to prove the following assertion. Suppose that $r, s \geqslant 2$ are integers, that $K$ is an ideal of a ring $R$ such that $R / K \cong M_{s}(D)$ for some ring $D$ and identify $M_{r}(R) / M_{r}(K)$ with $M_{r s}(D)$. Let $T$, or $T_{1}$, be the subring of $M_{r}(R)$, containing $M_{r}(K)$, and such that $T / M_{r}(K)$ is the ring of $r s \times r s$ upper, or lower respectively, triangular matrices over $D$. Then $T \cong T_{1}$.

As remarked earlier, Theorem 3.3 may be regarded as an extension of Schanuel's Lemma. We next make this connection explicit. Suppose that one assumes, in Theorem 3.3 , that each $\bar{P}_{i}$ has one extra summand, say $\bar{P}_{i} \cong$ $U^{(n(i))} \oplus V_{i}$. Let $\bar{\alpha}$ be a signed permutation of determinant +1 of $\bigoplus \bar{P}_{i}$ which permutes the copies of $U$ while leaving unaffected each $V_{i}$. Then a minor modification to the proof of Theorem 3.3 shows that one can lift $\bar{\alpha}$ to an automorphism of $\bigoplus P_{i}$. The simplest form of this generalization is given by the next lemma. (Note that, in the case $V_{i}=0$, this result does yield Schanuel's Lemma.)

3.7. Lemma (Schanuel's Lemma-stronger form). Consider the following short exact sequences of $R$-modules, for some ring $R$ :

$$
\begin{aligned}
& 0 \rightarrow K_{1} \rightarrow P_{1} \rightarrow U_{1} \oplus V_{1} \rightarrow 0, \\
& 0 \rightarrow K_{2} \rightarrow P_{2} \rightarrow U_{2} \oplus V_{2} \rightarrow 0,
\end{aligned}
$$

where $P_{1}$ and $P_{2}$ are projective and $U_{1} \cong U_{2}$. Let $A_{i}$ and $B_{i}$ denote the inverse images in $P_{i}$ of $U_{i}$ and $V_{i}$ respectively. Then there is an automorphism $\theta$ of $P_{1} \oplus P_{2}$ such that $\theta\left(K_{1} \oplus K_{2}\right)=K_{1} \oplus K_{2}$, while $\theta\left(P_{1} \oplus K_{2}\right)=B_{1} \oplus A_{2}$ and $\theta\left(K_{1} \oplus P_{2}\right)=$ $A_{1} \oplus B_{2}$.

Proof. Note that $\left(P_{1} \oplus P_{2}\right) /\left(K_{1} \oplus K_{2}\right) \cong U_{1} \oplus V_{1} \oplus U_{2} \oplus V_{2}$. Now, let $\bar{\theta}$ be the signed transposition $\langle 1,3\rangle$ of $U_{1} \oplus V_{1} \oplus U_{2} \oplus V_{2}$ which interchanges $U_{1}$ and $U_{2}$. As before,

$$
\langle 1,3\rangle=E_{13}(1) E_{31}(-1) E_{13}(1)
$$

and each of these three transvections is, in turn, an element of $E\left(P_{1} / K_{1}, P_{2} / K_{2}\right)$. Thus, Lemma 3.1 implies that there is an automorphism $\theta$ of $P_{1} \oplus P_{2}$ which induces $\bar{\theta}$. It is clear that $\theta$ has the desired properties.

The remainder of this section will consider consequences of Lemma 3.7. Recall that if $K$ is a right ideal of a ring $R$ then its idealizer ring is defined to be

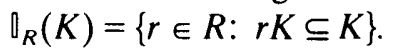

3.8. Corollary. Let $A, B$ be right ideals of a ring $R$ that are comaximal and coisomorphic; that is, $A+B=R$ and $R / A \cong R / B$. 
(i) There is an automorphism $\theta$ of $R^{(2)}$ which induces an isomorphism $A \oplus A \cong A \oplus B$. Consequently, given any four coisomorphic and comaximal right ideals, $A, B, C$ and $D$, then $A \oplus B \cong C \oplus D$.

(ii) For all $n \geqslant 2$ there exists an automorphism $\psi$ of $R^{(n)}$ which induces an isomorphism $A^{(n)} \cong B^{(n)}$.

Proof. Let $K=A \cap B$, so $R / K \cong R / A \oplus R / B$. Set $P_{1}=P_{2}=R$ and $K_{1}=K_{2}=K$. Consider the final isomorphism $K_{1} \oplus P_{2} \cong A_{1} \oplus B_{2}$ of Lemma 3.7. If we define $A=A_{1}=A_{2}$ and $B=B_{1}=B_{2}$, then this implies that $K \oplus R \cong A \oplus B$. Conversely, if we define $A=A_{1}=B_{2}$ and $B=B_{2}=A_{1}$, then $K \oplus R \cong A \oplus A$. The rest is clear.

In the proof of the last result, the easiest way to think of the two applications of Lemma 3.7 is that the first 'interchanges the two copies of $R / A$ in $P_{1} / K_{1} \oplus P_{2} / K_{2}$ ', while the second 'interchanges one copy of $R / B$ with a copy of $R / A$ '.

Remarks. If the module $R / A$ is Artinian, or even semiprimary, then Corollary 3.8 can be considerably strengthened: by [14, Theorem 1.5] any two presentations of $R / A$ by $R^{(2)}$ are isomorphic to each other; that is, some automorphism of $R^{(2)}$ carries one kernel onto the other.

Curiously, there are abundant examples of domains $R$ with right ideals $A$ and $B$ that satisfy the hypotheses of Corollary 3.8 but for which $A \neq B$. An easy example is given by the Weyl algebra $R=k\{y, x\} /(x y-y x-1)$. Then [20, Corollary 1.7] implies that $A=y R$ and $B=y^{2} R+(y x-1) R$ are coisomorphic, maximal right ideals of $R$ such that $B$ is not cyclic. Thus, $B \not A$. On the other hand (particularly when $R$ is not a domain) there are many circumstances where this situation cannot occur. For example, suppose that $R=M_{n}(S)$, for some ring $S$ and integer $n>1$ or that $R$ is a simple ring with a non-trivial idempotent $e$. Then any coisomorphic, maximal right ideals $A$ and $B$ of $R$ are actually isomorphic. This follows from [14, Theorem 1.5] in much the same way that Corollary 3.8 follows from Lemma 3.7.

3.9. Corollary. Let $A$ and $B$ be right ideals of a ring $R$ that are comaximal and coisomorphic.

(i) For all $n \geqslant 2, M_{n}(\mathrm{D}(A)) \cong M_{n}(\mathrm{D}(B))$.

(ii) If $n=2$ and $K=A \cap B$, then both are isomorphic to

$$
T=\left(\begin{array}{cc}
\llbracket(K) & K \\
R & R
\end{array}\right) .
$$

(iii) Moreover,

$$
\left(\begin{array}{cc}
\square(A) & A \\
R & R
\end{array}\right) \cong\left(\begin{array}{cc}
\square(B) & B \\
R & R
\end{array}\right) .
$$

Proof. (i) We use the automorphism $\psi$ provided by Corollary 3.8(ii). Note that

$$
M_{n}(\mathbb{Q}(A))=\left\{\alpha \in M_{n}(R): \alpha\left(A^{(n)}\right) \subseteq A^{(n)}\right\} .
$$

Since $\psi\left(A^{(n)}\right)=B^{(n)}$, it follows immediately that $\psi M_{n}(\mathbb{Q}(A)) \psi^{-1}=M_{n}(\mathbb{Q}(B))$.

(ii) If $n=2$, then Lemma 3.7 provides an automorphism $\theta$ of $R \oplus R$ such that 
$\theta(K \oplus R)=A \oplus A$. As in the proof of part (i), this induces an isomorphism $T \cong M_{2}(\square(A))$.

(iii) In this case, Lemma 3.7 provides an automorphism $\theta$ of $R \oplus R$ such that $\theta(A \oplus R)=B \oplus R$. Now, mimic the proof of part (i). Note that this part of the corollary does not require $A$ and $B$ to be comaximal.

Part (i) of Corollary 3.9 extends [4, Theorem 3.2], while examples in [4] also show that, in general, $\square(A) \neq \square(B)$. It is an easy exercise to use part (ii) of Corollary 3.9 to answer Chatters' question (1.2). The details are left to the reader since another solution of this question will follow from the results in $\S 4$ (see, in particular, (4.7)).

\section{Hidden by permutations}

Our first hidden matrix theorems uncover some $n \times n$ matrix subrings of $M_{n}(R)$ that are hidden by a permutation automorphism of an $n^{2} \times n^{2}$ homomorphic image of $M_{n}(R)$. In particular we prove Theorem 1.3 and Theorem 1.4 of the Introduction. Since these involve some complicated notation, we precede them by a simple example.

\subsection{Example. Consider the ring}

$$
T=\left(\begin{array}{ll}
R & I^{\prime} \\
J^{\prime} & R
\end{array}\right) \subseteq M_{2}(R),
$$

where $I^{\prime}, J^{\prime}$ are ideals of a ring $R$. Suppose that there is a surjective ring homomorphism $\phi: R \rightarrow M_{2}(D)$ for some ring $D$. Then $\phi\left(I^{\prime}\right)=M_{2}(I)$ and $\phi\left(J^{\prime}\right)=M_{2}(J)$ for appropriate ideals $I, J$ of $D$. Therefore $\phi_{2}$, the map that applies $\phi$ coordinatewise, maps $T$ onto the ring

$$
\bar{T}=\left(\begin{array}{cc}
M_{2}(D) & M_{2}(I) \\
M_{2}(J) & M_{2}(D)
\end{array}\right) \subseteq M_{4}(D) .
$$

Thus, the elements of $\bar{T}$ are those matrices of the form $\bar{t}$ shown in (4.1.3),

$$
\bar{t}=\left(\begin{array}{ccccc}
x_{11} & x_{12} & \vdots & i_{11} & i_{12} \\
x_{21} & x_{22} & \vdots & i_{21} & i_{22} \\
\ldots & \ldots & . & \ldots & \ldots \\
j_{11} & j_{12} & \vdots & y_{11} & y_{12} \\
j_{21} & j_{22} & \vdots & y_{21} & y_{22}
\end{array}\right), \quad \bar{t}^{\prime}=\left(\begin{array}{ccccc}
x_{11} & i_{11} & \vdots & x_{12} & i_{12} \\
j_{11} & y_{11} & \vdots & j_{12} & y_{12} \\
\ldots & \ldots & \cdot & \ldots & \ldots \\
x_{21} & i_{21} & \vdots & x_{22} & i_{22} \\
j_{21} & y_{21} & \vdots & j_{22} & y_{22}
\end{array}\right)
$$

where each $x_{\mu v}, y_{\mu v} \in D, i_{\mu v} \in I$ and $j_{\mu v} \in J$. Interchanging rows 2 and 3 , and also columns 2 and 3, of $\bar{t}$ yields the matrix $\bar{t}^{\prime}$ in (4.1.3). Thus, letting $\omega$ be the $4 \times 4$ permutation matrix $(2,3)$ we have:

$$
\omega \bar{T} \omega^{-1}=M_{2}(\bar{S}) \subseteq M_{4}(D) \quad \text { where } \quad \bar{S}=\left(\begin{array}{ll}
D & I \\
J & D
\end{array}\right)
$$

Thus $\bar{T}$ is permutation isomorphic to a $2 \times 2$ matrix ring in $M_{4}(D)$. If $I^{\prime} \neq R$, then 
$T$ is clearly not permutation isomorphic to a $2 \times 2$ matrix ring in $M_{2}(R)$. However, our first hidden matrix theorem shows that, if $I^{\prime}$ and $J^{\prime}$ contain $\operatorname{ker} \phi$, then $T$ is conjugate to a $2 \times 2$ matrix ring in $M_{2}(R)$.

4.2. THEOREM. Let $\phi: R \rightarrow M_{n}(D)$ be a surjective ring homomorphism with kernel $K$, and $\phi_{n}: M_{n}(R) \rightarrow M_{n^{2}}(D)$ the map that applies $\phi$ coordinatewise. Let $T$ be a ring such that $M_{n}(K) \subset T \subseteq M_{n}(R)$ and such that $\phi_{n}(T)$ is signed permutation isomorphic to an $n \times n$ matrix ring in $M_{n^{2}}(D)$; that is,

$$
\omega \phi_{n}(T) \omega^{-1}=M_{n}(\bar{S})
$$

with $\omega \in S_{n^{2}}^{ \pm}(D)$ and $\bar{S} \subseteq \phi(R)=M_{n}(D)$. Assume, further, that either

(i) $\phi_{n}(T)$ is a tiled subring of $M_{n^{2}}(D)$; or

(ii) $Z(R)$, the centre of $R$, contains an $n$th root of -1 (this always holds if $n$ is odd); or

(iii) $\operatorname{det} \omega=1$.

Then for some unit $\alpha$ of $M_{n}(R)$ we have $T \cong \alpha T \alpha^{-1}=M_{n}(S)$ where $S$ is the ring defined by $K \subset S \subseteq R$ and $\phi(S)=\bar{S}$.

Proof. We can suppose that $n \geqslant 2$. We first prove the theorem assuming that either (ii) or (iii) holds. In either of these situations, Corollary 3.4 shows that $\omega$ can be lifted to a unit $\alpha$ in $M_{n}(R)$; thus $\phi_{n}(\alpha)=\omega$. We show that $\alpha T \alpha^{-1}=M_{n}(S)$ as claimed. Since $M_{n}(K)$ is an ideal of $M_{n}(R)$, we have $\alpha M_{n}(K) \alpha^{-1}=M_{n}(K)$. Therefore $\alpha T \alpha^{-1} \supseteq M_{n}(K)$, and so $\alpha T \alpha^{-1}$ is the full inverse image, under $\phi_{n}$, of $M_{n}(\bar{S})$. Let $S$ be as described in the theorem. Then $M_{n}(S) \supseteq M_{n}(K)$ and $\phi_{n}\left(M_{n}(S)\right)=M_{n}(\bar{S})=\phi_{n}\left(\alpha T \alpha^{-1}\right)$. Therefore $\alpha T \alpha^{-1}=M_{n}(S)$ as claimed.

Finally, suppose situation (i) holds, with $\operatorname{det} \omega=-1$. Let $\omega^{\prime}$ be a matrix obtained by changing the sign of one of the non-zero entries of $\omega$. Since $\omega$ is a signed permutation matrix, it has exactly one non-zero entry in each row and column and so det $\omega^{\prime}=-\operatorname{det} \omega=+1$. Since $\phi_{n}(T)$ is a tiled subring of $M_{n^{2}}(D)$, the ring $\phi_{n}(T)$ is unchanged if we multiply one of its $n^{2}$ rows or columns by -1 . Therefore $\omega^{\prime} \phi_{n}(T)\left(\omega^{\prime}\right)^{-1}=\omega \phi_{n}(T) \omega^{-1}$. Thus, after replacing $\omega$ by $\omega^{\prime}$ in (4.2.1), we are in the situation already considered.

Remark. We caution the reader that conditions (i)-(iii) of the theorem cannot be deleted (see Corollary 6.8). Moreover, when (i) or (ii) holds, (4.2.1) cannot be replaced by the simpler statement that $\phi_{n}(T)$ is isomorphic to an $n \times n$ matrix ring (see Corollary 5.16).

4.3. Definition. Let $D$ be a ring and $r, s$ positive integers; and note that we have the identifications $M_{r}\left(M_{s}(D)\right)=M_{r s}(D)=M_{s}\left(M_{r}(D)\right)$. These identifications lead naturally to a permutation automorphism of $M_{r s}(D)$, which is described as follows.

Let $N_{r s}=\{1,2,3, \ldots, r s\}$. We can view $N_{r s}$ as consisting of $r$ blocks, each with $s$ consecutive integers, or as consisting of $s$ blocks, each with $r$ consecutive integers. Let $\Omega$ be the permutation of $N_{r s}$ that sends

$i$ th integer in $\mu$ th $s$-integer block $\rightarrow \mu$ th integer in $i$ th $r$-integer block. 
In other words,

$$
\Omega(i+(\mu-1) s)=\mu+(i-1) r .
$$

Let $\omega$ be the $r s \times r s$ permutation matrix such that left multiplication by $\omega$ performs the row-permutation $\Omega$ on $M_{r s}(D)$. Then the inner automorphism $\psi: C \rightarrow \omega C \omega^{-1}$ of $M_{r s}(D)$ performs the permutation $\Omega$ on both the rows and columns of all elements $C$ of $M_{r s}(D)$.

In the situation described in Example 4.1, conjugation by $\omega$ becomes the map $\bar{t} \rightarrow \bar{t}^{\prime}$ in (4.1.3).

4.4. Lemma. Fix integers $r, s \geqslant 1$. Let $\bar{S}=\left(D_{i j}\right)$ be a tiled subring of $M_{r}(D)$ for some ring $D$, and let

$$
\bar{T}=\left(\begin{array}{cccc}
M_{s}\left(D_{11}\right) & M_{s}\left(D_{12}\right) & \cdots & M_{s}\left(D_{1 r}\right) \\
M_{s}\left(D_{21}\right) & M_{s}\left(D_{22}\right) & \cdots & M_{s}\left(D_{2 r}\right) \\
\vdots & \vdots & \ddots & \vdots \\
M_{s}\left(D_{r 1}\right) & M_{s}\left(D_{r 2}\right) & \cdots & M_{s}\left(D_{r r}\right)
\end{array}\right) \subseteq M_{r s}(D) .
$$

(i) We have $\omega \bar{T} \omega^{-1}=M_{r}(\bar{S})$ where $\omega$ is the permutation matrix in Definition 4.3.

(ii) If $r=s$, and $s \equiv 0,1(\bmod 4)$, then $\operatorname{det} \omega=1$.

Proof. (i) Let $\bar{T}^{\prime}=\omega \bar{T} \omega^{-1}$. Subdivide $\bar{T}^{\prime}$ into $r \times r$-blocks, and choose a particular position within one of these blocks, say its $(i, j)$-entry. It suffices to prove that this $(i, j)$-entry is $D_{i j}$ regardless of which $r \times r$-block we choose (since the entries of $\bar{T}^{\prime}$ are formed by permuting the entries of $\bar{T}$ ).

Now consider, say, the $(\mu, v)$-block of $\bar{T}^{\prime}$, of size $r \times r$. By (4.3.1) we see that the $(i, j)$-entry of this $(\mu, v)$-block of $\bar{T}^{\prime}$ equals the $(\mu, v)$-entry of the $(i, j)$-block of $\bar{T}$ of size $s \times s$. By (4.4.1) we see that all entries of this $(i, j)$-block of $\bar{T}$ equal $D_{i j}$. Thus statement (i) holds.

(ii) It suffices to show that the permutation $\Omega$ defined in (4.3) is a product of an even number of transpositions. When $r=s$ it follows from formula (4.3.2) that $\Omega$ is the product of all distinct (and necessarily disjoint) transpositions of the form

$$
(i+(\mu-1) s, \mu+(i-1) s) .
$$

We have $i+(\mu-1) s=\mu+(i-1) s$ if and only if $i=\mu$. Now, the total number of ordered pairs of the form (4.4.2) is $s^{2}$; and by the previous sentence $s^{2}-s$ of these are transpositions. Since transpositions are unordered pairs, the number of distinct transpositions of the form (4.4.2) is $\frac{1}{2} s(s-1)$, which is an even integer if $s \equiv 0,1(\bmod 4)$.

We now state our second hidden matrix theorem, the most general form we know of the basic situation illustrated in Example 4.1.

4.5. TheOREM. Let $\phi: R \rightarrow M_{n}(D)$ be a surjective ring homomorphism with kernel $K$, and let $T=\left(R_{i j}\right)$ be a tiled subring of $M_{n}(R)$ such that:

(i) every tile $R_{i j} \supseteq K$; and

(ii) for every diagonal tile $R_{i i}$, the ring $\phi\left(R_{i i}\right)$ contains the standard $n \times n$ matrix units $e_{i j}$ of $M_{n}(D)$.

Then $T \cong M_{n}(S)$, where the ring $S$ is defined in (4.5.2) below. 
Proof. First we show that $\phi_{n}(T)$ is a tiled subring of $M_{n^{2}}(D)$. Each tile $R_{i j}$ is an $\left(R_{i i}, R_{i j}\right)$-bimodule contained in $R$. Therefore, each $\phi\left(R_{i j}\right)$ is a $\left(\phi\left(R_{i i}\right), \phi\left(R_{j j}\right)\right)$ bimodule contained in $\phi(R)=M_{n}(D)$, and so $\phi\left(R_{i j}\right)$ is closed under left and right multiplication by the matrix units $e_{i j}$ given by hypothesis (ii). This implies that, for each $i$ and $j, \phi\left(R_{i j}\right)=M_{n}\left(D_{i j}\right)$ for additive subgroups $D_{i j}=e_{11} \phi\left(R_{i j}\right) e_{11} \subseteq D$. Thus $\phi_{n}(T)$ is the following tiled subring of $M_{n^{2}}(D)$ :

$$
\phi_{n}(T)=\left(\begin{array}{cccc}
M_{n}\left(D_{11}\right) & M_{n}\left(D_{12}\right) & \cdots & M_{n}\left(D_{1 n}\right) \\
M_{n}\left(D_{21}\right) & M_{n}\left(D_{22}\right) & \cdots & M_{n}\left(D_{2 n}\right) \\
\vdots & \vdots & \ddots & \vdots \\
M_{n}\left(D_{n 1}\right) & M_{n}\left(D_{n 2}\right) & \cdots & M_{n}\left(D_{n n}\right)
\end{array}\right) \subseteq M_{n^{2}}(D) .
$$

In particular, Lemma 4.4 shows that $\phi_{n}(T)$ is permutation isomorphic to $M_{n}(\bar{S})$, for $\bar{S}=\left(D_{i j}\right) \subseteq M_{n}(D)$. Finally, this implies that all the hypotheses of Theorem 4.2(i) are satisfied and so $T \cong M_{n}(S)$, where $S$ is defined by

$$
K \subset S \subseteq R \quad \text { and } \quad \phi(S)=\bar{S}=\left(D_{i j}\right)
$$

Remarks. One would like to replace the awkward condition (ii) of Theorem 4.5 by the simpler condition that each $\phi\left(R_{i i}\right)$ is an $n \times n$ matrix ring. However, this generalization is false, as will be shown in Corollary 5.16. One situation in which condition (ii) can be deleted is given by Theorem 1.3 of the introduction of this paper:

4.6. Corollary. Let $K$ be an ideal of a ring $R$ and suppose that $T=\left(R_{i j}\right)$ is a tiled subring of $M_{n}(R)$ containing $M_{n}(K)$. Suppose that $R_{i i}=R_{11}$ for all $i$ and that $R_{11} / K \cong M_{n}\left(D^{\prime}\right)$, for some ring $D^{\prime}$. Then $T \cong M_{n}(S)$ for some ring $S$ (described in the proof).

Proof. Since $R_{11} / K \cong M_{n}\left(D^{\prime}\right)$, the ring $R_{11} / K$ contains a set $\left\{e_{j k}\right\}$ of $n \times n$ matrix units. These matrix units are contained in the ring $R / K \supseteq R_{11} / K$, and so $R / K$ is an $n \times n$ matrix ring, by Lemma 2.1. Choose a corresponding matrix representation; that is, an identification $R / K=M_{n}(D)$, in which the $e_{j k}$ become the standard matrix units. This yields a surjective ring homomorphism $\phi: R \rightarrow$ $M_{n}(D)$ with kernel $K$. Since $R_{11} / K=R_{i i} / K$ for every $i$, hypothesis (ii) of Theorem 4.5 is satisfied, and so $T \cong M_{n}(S)$ for the ring $S$ defined by (4.5.2).

4.7. Simple examples. The next few examples illustrate our hidden matrix theorems in some of their easiest guises.

(i) Let $K$ be any ideal of a ring $R^{\prime}$ such that $R^{\prime} / K=M_{n}\left(D^{\prime}\right)$ for some ring $D^{\prime}$, and let $T^{\prime}=\operatorname{diag}\left(R^{\prime}, R^{\prime}, \ldots, R^{\prime}\right)+M_{n}(K)$ (that is, all diagonal tiles equal $R^{\prime}$, while all off-diagonal tiles equal $K)$. Then $T^{\prime} \cong M_{n}\left(S^{\prime}\right)$ for some ring $S^{\prime}$. Moreover, by Theorem 4.5, we can take $S^{\prime}$ to be the subring of $R^{\prime}$, containing $K$, such that $S^{\prime} / K=\operatorname{diag}\left(D^{\prime}, D^{\prime}, \ldots, D^{\prime}\right)$.

(ii) The ring $T^{\prime}$ in part (i) is the simplest example afforded by Theorem 4.5, but it immediately implies a large part of the general case. For, any ring $T$ that satisfies the hypotheses of the theorem will contain the ring $T^{\prime}$ described above: replace every off-diagonal tile of $T$ (which contains $K$, by hypothesis) by $K$, and replace every diagonal tile $R_{i i}$ by $R^{\prime}=\bigcap_{i} R_{i i}$. The critical fact here is that, by hypothesis (ii) of the theorem, $R^{\prime} / K$ still contains the standard matrix units $e_{i j}$. 
Therefore $T^{\prime} \subseteq T$. By part (i), $T^{\prime}$ is an $n \times n$ matrix ring. Hence, by Lemma 2.1, so is the larger ring $T$, say $T \cong M_{n}(S)$. The disadvantage of this approach to the problem is that it does not seem to provide an explicit description of the ring $S$.

(iii) Theorem 4.2 can be applied to show that certain non-tiled subrings of $M_{n}(R)$ are hidden matrix rings. Let $K$ be an ideal of a ring $R$ such that $R / K$ is isomorphic to a $2 \times 2$ matrix ring, say $\phi(R)=M_{2}(D)$ with $\operatorname{ker}(\phi)=K$. Identify $R$ with the set of scalar matrices in $M_{2}(R)$ and set

$$
T=R+M_{2}(K)=\left\{\left(\begin{array}{rr}
r+k_{1} & k_{2} \\
k_{3} & r+k_{4}
\end{array}\right): r \in R \text { and } k_{j} \in K\right\},
$$

a subring of $M_{2}(R)$. Consider the matrices, in $M_{4}(D)$, of the forms:

$$
\alpha=\left(\begin{array}{llll}
a & b & 0 & 0 \\
c & d & 0 & 0 \\
0 & 0 & a & b \\
0 & 0 & c & d
\end{array}\right) \text { and } \beta=\left(\begin{array}{cccc}
a & 0 & b & 0 \\
0 & a & 0 & b \\
c & 0 & d & 0 \\
0 & c & 0 & d
\end{array}\right)
$$

Note that $\bar{T}=\phi_{2}(T)$ consists of all matrices $\alpha \in M_{4}(D)$ of the form $\alpha$ displayed in (4.7.2), and so $\bar{T} \cong M_{2}(D)$. In fact $\bar{T}$ is permutation-isomorphic, in $M_{4}(D)$, to the $2 \times 2$ matrix ring $M_{2}\left(D \cdot I_{2}\right)$, because the matrices in (4.7.2) are related by $\omega \alpha \omega^{-1}=\beta$ where $\omega$ is the permutation matrix corresponding to the permutation $(2,3)$. Thus, Theorem 4.2(ii) implies that

$$
\text { if } \vee-1 \in Z(R) \text { then } T \text { is a full } 2 \times 2 \text { matrix ring. }
$$

Strangely, if $\mathrm{V}-1 \notin Z(R)$, there exist examples where $T$ is and where $T$ is not a full $2 \times 2$ matrix ring (see $\S 6$ ). Moreover, for any such exceptional $T, M_{2}(T)$ is a $4 \times 4$ matrix ring. This, and a number of other facts about the $n \times n$ analogue of the above situation, are contained in the following theorem.

4.8. THEOREM. Let $K$ be an ideal of a ring $R$ such that $R / K \cong M_{n}(D)$, for some ring $D$. Identify $R$ with the scalar matrices in $M_{n}(R)$ and set $T=R+M_{n}(K)$.

(i) If either $n \equiv 0,1,3(\bmod 4)$ or $Z(R)$ contains a square root of -1 , then $T$ is an $n \times n$ matrix ring.

(ii) For all $n, M_{n}(T)$ is an $n^{2} \times n^{2}$ matrix ring. Moreover, for all $n$ and all even $k$, there exists a ring $S$ such that $M_{k}(T) \cong M_{k n}(S)$.

Remark. The ring $S$ is easy to describe. Choose any surjective ring homomorphism $\phi: R \rightarrow M_{n}(D)$ with $\operatorname{ker} \phi=K$. Then $S=\phi^{-1}\left(D \cdot I_{n}\right)$ where $D \cdot I_{n}$ denotes the set of scalar matrices in $M_{n}(D)$.

Proof. (i) Let $\phi$ and $S$ be as in the Remark, and for consistency with the notation of Theorem 4.2 let $\bar{S}=\phi(S)$, the set of scalar matrices in $M_{n}(D)$. Let $\bar{T}=\phi_{2}(T)$. We claim that

$$
\omega \bar{T} \omega^{-1}=M_{n}\left(D \cdot I_{n}\right)=M_{n}(\bar{S}),
$$

where $\omega$ is the permutation matrix in Definition 4.3 (with $r=s=n$ ). 
To prove this, consider the block form of an element $\bar{t} \in \bar{T}$, say $\bar{t}=$ $\operatorname{diag}(\bar{r}, \bar{r}, \ldots, \bar{r})$ where each of the $n$ equal diagonal blocks $\bar{r}$ is an $n \times n$ matrix over $D$ with $(\mu, v)$-entry $\bar{r}_{\mu v}$. Let $\bar{t}^{\prime}=\omega \bar{t} \omega^{-1} \in M_{n^{2}}(D)$. Subdivide $\bar{t}^{\prime}$ into $n \times n$ blocks, and choose one block, say its $(\mu, v)$-block $x$. It suffices to show that $x=\bar{r}_{\mu v} \cdot I_{n}$. First consider an off-diagonal entry $x_{i j}$ of $x$; thus $i \neq j$. By (4.3.1), $x_{i j}$ is the $(\mu, v)$-entry of the $(i, j)$-block of $\bar{t}$. The given block-diagonal form $\bar{t}=$ $\operatorname{diag}(\bar{r}, \bar{r}, \ldots, \bar{r})$ of $\bar{t}$ shows that its off-diagonal $(i, j)$-block is zero, and hence $x_{i j}=0$. Next consider a diagonal entry $x_{i i}$ of $x$. This equals the $(\mu, v)$-entry of the $(i, i)$-block of $\bar{t}$. All diagonal blocks of $\bar{t}$ equal $\bar{r}$. Hence $x_{i i}=\bar{r}_{\mu v}$ for all $i$. This completes the proof of the claim.

We now consider several cases. First consider the cases where $n \equiv 1$ or $3(\bmod 4)$; or $n \equiv 2(\bmod 4)$, and $Z(R)$ contains a square root of -1 . In any of these situations, $Z(R)$ contains an $n$th root of -1 . (If $n$ is odd, -1 is itself such a root; and if $n \equiv 2(\bmod 4)$, any square root of -1 is an $n$th root.) Therefore part (i) of the present theorem and the supplementary Remark follow from Theorem 4.2(ii). Finally, consider the case $n \equiv 0(\bmod 4)$. In this case Lemma 4.4(ii) shows that det $\omega=1$, so Theorem 4.2(iii) completes the proof.

(ii) If $n$ is odd, then $T \cong M_{n}(S)$, so the desired result is obvious. Thus, we may assume that $n$ is even, in which case it suffices to prove the second assertion. Let $\omega$ be as in (4.8.1), and let $\beta$ be the direct sum of $k$ copies of $\omega$. That is, let $\beta \in M_{k n^{2}}(D)$ be the block-diagonal matrix whose $k$ diagonal blocks all equal $\omega$. Since $k$ is even and $\operatorname{det} \omega= \pm 1$, we have $\operatorname{det} \beta=1$. Moreover, since (4.8.1) holds, we have $\beta M_{k}(\bar{T}) \beta^{-1}=M_{k n}(\bar{S})$. By Corollary 3.4(ii), with $r$ replaced by $k n$ and $s$ replaced by $n$, one sees that $\beta$ lifts to a unit of $M_{k n}(R)$. The result now follows as in the proof of Theorem 4.2 .

The following consequence of 4.7(i) is a partial generalization of Theorem 4.5 to the situation in which $K$ is only assumed to be a one-sided, rather than two-sided, ideal.

4.9. Corollary. Let $K$ be a right ideal of a ring $R$ such that $R / K \cong U^{(n)}$, for some module $U$. Let $T=\left(R_{i j}\right)$ be a tiled subring of $M_{n}(R)$, containing $M_{n}(K)$, and such that $R_{i i} \supseteq \mathbb{D}_{R}(K)$ for all $i$. Then $T \cong M_{n}(S)$ for some ring $S$.

Proof. By 4.7(i) the subring $T^{\prime}=\operatorname{diag}\left\{\mathbb{0}_{R}(K), \ldots, \mathbb{0}_{R}(K)\right\}+M_{n}(K)$ of $T$ is an $n \times n$ matrix ring. Now use Lemma 2.1 .

\section{Abstract problem, in dimension 1}

For the motivating problem of this section, consider Noetherian prime rings $T \subset M_{n}(R)=M$ of (Rentschler-Gabriel) Krull dimension 1, that satisfy the hypotheses of Question 1.1. We obtain strong structure theorems for such a ring $T$; for example,

$$
T \cong \operatorname{End}_{S}\left(S^{n-1} \oplus L\right),
$$

for an appropriate right ideal $L$ of a ring $S$. Moreover, $T$ is a tiled matrix subring of $M$, for some matrix presentation $M=M_{n}(\widetilde{R})$ (see Corollary 5.9). This also allows us to produce counter-examples to several questions raised in the previous section. 
The key tools in this section are the following concepts of genus and $n$-genus rings. The latter concept generalizes that of being an $n \times n$ matrix ring but has the advantage that it descends to subrings in a way that is false for the property of being a matrix ring. This, in turn, means that results like (5.0.1) can be proved for considerably more general rings than those mentioned in the last paragraph; see, in particular, Theorems 5.6 and 5.8.

5.1. Definition. Let $V$ be a right module over a right Noetherian ring $A$ and $I$ a prime ideal of $A$. Write $Q(A / I)$ for the simple Artinian ring of fractions of $A / I$. Then, the reduced rank of $V$ at $I$ is defined to be

$$
\rho(V, I)=\text { length }_{Q(A / I)} \widetilde{V}_{l}, \quad \text { where } \widetilde{V}_{I}=\left(V / V I \otimes_{A / I} Q(A / I)\right) .
$$

Note that $\rho(, I)$ is additive on direct sums. If $P$ and $Q$ are two finitely generated projective right $A$-modules, then $P$ is said to be in the genus of $Q$ if $\rho(Q, I)=\rho(P, I)$ for every prime ideal $I$ of $A$. Finally, a right Noetherian $\operatorname{ring} A$ that is the direct sum of $n$ right ideals $P_{1}, \ldots, P_{n}$, all in the same genus, will be called a right $n$-genus ring with decomposition $A=P_{1} \oplus \ldots \oplus P_{n}$.

We begin with some observations to illustrate these concepts. Since these facts will not be needed subsequently, the proofs are only sketched. If $A$ is a finitely generated module over a central, Noetherian subring $C$, then our notion of genus does coincide with the classical concept. More precisely, two finitely generated, projective right $A$-modules $P$ and $Q$ are in the same genus if and only if $P_{\mathbf{m}} \cong Q_{\mathbf{m}}$ as $A_{\mathbf{m}}$-modules for every maximal ideal $\mathbf{m}$ of $C$. (To see this, use Lemma 5.2, below.) Clearly, any right Noetherian $n \times n$ matrix ring is a right $n$-genus ring. A less trivial example is given by any Dedekind prime ring $A$ of uniform dimension $n$. (To prove this, use [15, Lemma 5.7.5] and [15, Theorem 5.7.10].) Finally, if $A$ is a Noetherian ring, then it is easy to show that $A$ is a right $n$-genus ring if and only if $A$ is a left $n$-genus ring. (Use the fact that, if $A=\oplus P_{j}$, for right ideals $P_{j}$ in the same genus, then $A=\bigoplus P_{j}^{*}$, where $P_{j}^{*}=\operatorname{Hom}\left(P_{j}, A\right)$ and check that $\rho\left(P_{j}, I\right)=$ $\rho\left(P_{j}^{*}, I\right)$ for every prime ideal $I$ of $A$.)

The first main result of this section will be a decomposition theorem for right Noetherian right $n$-genus rings, analogous to (5.0.1). We begin with some easy preliminary results. A ring $A$ is called semilocal if $A / J(A)$ is semisimple Artinian, where $J(A)$ denotes the Jacobson radical of $A$.

5.2. Lemma. (i) Suppose that $P$ and $Q$ are finitely generated, projective right modules over a semilocal, right Noetherian ring $B$ such that $\rho(P, I)=\rho(Q, I)$ for every maximal ideal I of $B$. Then, $P \cong Q$.

(ii) In particular, a semilocal, right Noetherian, right $n$-genus ring is an $n \times n$ matrix ring.

Proof. In order to prove part (i), note that $\rho(P, I)=\rho(Q, I)$ if and only if $\widetilde{Q}_{l} \cong \widetilde{P}_{l}$, in the notation of (5.1). Thus, by hypothesis, $P / P M=$ $\widetilde{P}_{M} \cong \widetilde{Q}_{M}=Q / Q M$, for every maximal ideal $M$ of $B$. Therefore, $P / P J(B) \cong$ $Q / Q J(B)$. As $P$ and $Q$ are projective, this isomorphim lifts to a surjection, and hence an isomorphism $P \Im Q$. Part (ii) is an immediate consequence of part (i) and Lemma 2.1 .

If $N$ is a module over a ring $A$, we will denote $\operatorname{Hom}_{A}(N, A)$ by $N^{*}$, provided 
that the notation is unambiguous. If $N$ is a projective right $A$-module, we will always identify $\operatorname{End}_{A}(N)$ with $N \otimes_{A} N^{*}$.

5.3. Lemma. (i) Let $A$ be a right Noetherian, right $n$-genus ring with decomposition $A=\bigoplus_{i=1}^{n} P_{i}$. If $N$ is a right $A$-module in the genus of $P_{1}$, then $N$ is a progenerator.

(ii) Let $W_{A}$ be a progenerator over a right Noetherian ring $A$ and set $S=W \otimes W^{*}=\operatorname{End}_{A}(W)$. Then finitely generated, projective right $A$-modules $P$ and $Q$ are in the same genus if and only if $P \otimes_{A} W^{*}$ and $Q \otimes W^{*}$ are in the same genus as right $S$-modules.

Proof. (i) By hypothesis, $N$ is projective. Let $I$ be a maximal ideal of $A$. As $\rho(, 0)$ is additive on direct sums, $\rho(N, I)=\rho\left(P_{1}, I\right)=(1 / n) \rho(A, I) \neq 0$. Thus there exists $\bar{\phi} \in \operatorname{Hom}_{A}(N / N I, A / I)$ with $\bar{\phi} \neq 0$. Since $N$ is projective, $\bar{\phi}$ lifts to $\phi \in N^{*}$ such that $\phi(N) \nsubseteq I$. Since $I$ is arbitrary, this implies that $N^{*} N=A$.

(ii) Suppose that $J$ is a prime ideal of $S$. Then $J=W \otimes_{A} I \otimes_{A} W^{*}$ for some prime ideal $I$ of $A$ and this provides a one-to-one correspondence between the prime ideals of $S$ and $A$ (see, for example, [15, Theorem 3.5.9)]. Moreover, by [15, Proposition 3.6.9], the rings of fractions $Q(S / J)$ and $Q(A / I)$ are Morita equivalent via the module

$$
\widetilde{W}^{*}=Q(A / I) \otimes_{A / I}\left(W^{*} / I W^{*}\right) \cong\left(W^{*} / W^{*} J\right) \otimes_{S / J} Q(S / J) .
$$

It now follows from [15, Lemma 3.5.8] that $\rho_{A}(P, I)=\rho_{S}\left(P \otimes W^{*}, J\right)$. This is equivalent to the assertion of the lemma.

Putting these results together gives:

5.4. Proposition. The following conditions on a right Noetherian ring $A$ are equivalent:

(i) $A$ is a right $n$-genus ring;

(ii) there exist a right Noetherian ring $S$ and right $S$-modules $L_{1}, \ldots, L_{n}$ in the genus of $S$ such that $A \cong \operatorname{End}_{S}\left(L_{1} \oplus \ldots \oplus L_{n}\right)$.

Proof. (i) $\Rightarrow$ (ii) Suppose that $A$ has decomposition $A=\bigoplus_{1}^{n} P_{i}$ and set $S=$ End $_{A}\left(P_{1}\right)$. By Lemma 5.3(i), $S$ is Morita equivalent to $A$ and, by Lemma 5.3(ii), each $P_{j} \otimes_{A} P_{1}^{*}$ is in the genus of $P_{1} \otimes_{A} P_{1}^{*} \cong S$. Finally, $A \cong \operatorname{End}_{S}\left(P_{1}^{*}\right) \cong$ $\operatorname{End}_{s}\left(\left(P_{1} \otimes P_{1}^{*}\right) \oplus \ldots \oplus\left(P_{n} \otimes P_{1}^{*}\right)\right)$, as required.

(ii) $\Rightarrow$ (i) By Lemma 5.3(i), $L=\bigoplus_{1}^{n} L_{j}$ is a right $S$-progenerator and so ${ }_{A} L_{S}$ provides a Morita equivalence between $S$ and $A$. Moreover, $A \cong L \otimes_{S} L^{*} \cong$ $\bigoplus_{i=1}^{n}\left(L_{i} \otimes_{S} L^{*}\right)$. Finally, Lemma 5.3(ii) implies that, as right $A$-modules, each $P_{i}=L_{i} \otimes_{S} L^{*}$ is the same genus.

In order to obtain results akin to (5.0.1) we need to assume that the $\operatorname{ring} A$ has (Rentschler-Gabriel) Krull dimension 1, written $\mathrm{Kdim} A=1$. (If $A$ is a finite module over a central, Noetherian subring $C$, then this is equivalent to demanding that $C$ have classical Krull dimension 1; see [15, Chapter 6].) The reason why Krull dimension 1 is helpful is that it enables one to prove the 
following strong decomposition results for modules in the same genus. These all follow from the non-commutative versions of Serre's Theorem and Bass's Cancellation Theorem, as described in $\S 5$ of [19]. One should remark that the results in $[19, \S 5]$ are all proved under the hypothesis that the ring be Noetherian. However, as is remarked in the introduction to [5], if one replaces [19, Theorem $3.1]$ by [5, Theorem 5.4] in the proofs of those results then they will also be valid for right Noetherian rings.

5.5. Proposition. Let $A$ be a right Noetherian ring with $\operatorname{Kdim}(A)=1$ and $X$ a finitely generated, projective right $A$-module.

(i) Suppose that $Y$ is an A-module in the genus of $X^{(s)}$, for some integer $s$. Then $Y \cong X^{(s-1)} \oplus X^{\prime}$ for some module $X^{\prime}$, necessarily in the genus of $X$.

(ii) Suppose that $A_{A}$ is in the genus of $X^{(n)}$, and let $Y$ be an A-module in the genus of $X^{(c n+r)}$ with $r$ strictly positive. Then $Y \cong A^{(c)} \oplus Y^{\prime}$ for some module $Y^{\prime}$ in the genus of $X^{(r)}$.

(iii) Assume that $X_{A}$ is a progenerator. Let $X_{1}, X_{2}$ and $X_{3}$ be projective, finitely generated right $A$-modules such that $X_{1} \oplus X_{3} \cong X_{2} \oplus X_{3}$ and $\rho\left(X_{1}, I\right) \geqslant 2 \rho(X, I)$ for all prime ideals I of $A$. Then $X_{1} \cong X_{2}$.

Proof. (i) Suppose that $W$ and $Z$ are two projective, finitely generated right $A$-modules and that $\rho(W, I) \geqslant 2 \rho(Z, I)$ for all prime ideals $I$ of $A$. Then [19, Corollary 5.10] implies that $W \cong Z \oplus Z^{\prime}$ for some module $Z^{\prime}$. Note that, as $\rho(, I)$ is additive on direct sums, $\rho\left(Z^{\prime}, I\right)=\rho(W, I)-\rho(Z, I)$ for all prime ideals $I$ of $A$. The result now follows by the obvious induction on $s$.

(ii) By part (i), $A \cong \bigoplus_{i=1}^{n} X_{i}$, where each $X_{i}$ is in the genus of $X$. An induction similar to that used in part (i) shows that $Y \cong X_{1}^{(c)} \oplus \ldots \oplus X_{n}^{(c)} \oplus Q^{\prime}$, for some $Q^{\prime}$.

(iii) In this case, we will use the following consequence of [19]. Let $S$ be a right Noetherian ring with $\operatorname{Kdim}(S)=1$. Suppose that $L_{1}$ and $L_{2}$ are two finitely generated, projective right $S$-modules such that $L_{1} \oplus S \cong L_{2} \oplus S$ and $\rho\left(L_{1}, I\right) \geqslant$ $2 \rho(S, I)$ for all prime ideals $I$ of $S$. Then [19, Corollary 5.11] implies that $L_{1} \cong L_{2}$. This is not quite general enough to prove part (iii) directly, but it can be applied after a change of rings.

Since $X$ is a progenerator, $X_{3} \oplus X_{4} \cong X^{(t)}$ for some integer $t$ and module $X_{4}$. We may therefore replace $X_{3}$ by $X^{(t)}$ and, by induction, it suffices to prove the result when $t=1$. Set $S=X \otimes_{A} X^{*}$ and $L_{j}=X_{j} \otimes_{A} X^{*}$. Thus, $L_{1} \oplus S \cong L_{2} \oplus S$. Moreover, the proof of Lemma 5.3(ii) implies that $\rho\left(L_{1}, J\right) \geqslant 2 \rho(S, J)$, for each prime ideal $J$ of $S$. Since $\operatorname{Kdim}(S)=1$, the last paragraph implies that $L_{1} \cong L_{2}$. Consequently, $X_{1} \cong L_{1} \otimes_{S} X \cong L_{2} \otimes X \cong X_{2}$.

Combined with Proposition 5.4, this proposition gives the desired version of (5.0.1):

5.6. Theorem. Let $T$ be a right Noetherian, right $n$-genus ring such that $\operatorname{Kdim} T=1$. Then there exist a ring $S$ Morita equivalent to $T$ and a projective, 
finitely generated right $S$-module $L$ in the genus of $S$ such that

$$
T \cong \operatorname{End}_{S}\left(S^{(n-1)} \oplus L\right) \cong\left(\begin{array}{ccccc}
S & S & \cdots & S & L^{*} \\
S & S & \cdots & S & L^{*} \\
\vdots & \vdots & \ddots & \vdots & \vdots \\
S & S & \cdots & S & L^{*} \\
L & L & \cdots & L & E
\end{array}\right)_{n \times n}
$$

Here, $L^{*}=\operatorname{Hom}_{S}(L, S)$ and $E=L \otimes_{S} L^{*}=\operatorname{End}_{S}(L)$.

Proof. Proposition 5.4 implies that there exist a ring $S$ Morita equivalent to $T$ and right $S$-modules $L_{j}$ in the genus of $S$ such that $T \cong \operatorname{End}_{s}\left(L_{1} \oplus \ldots \oplus L_{n}\right)$. By Proposition 5.5(i), $\bigoplus_{j=1}^{n} L_{j} \cong S^{(n-1)} \oplus L$, for some module $L$ in the genus of $S$.

Recall that any non-commutative Dedekind prime ring $T$ of uniform dimension $n$ is an $n$-genus ring and so Theorem 5.6 applies. However, in this case, the conclusion of the theorem is well-known (see, for example, [15, Corollary 5.7.9]) but, even here, $T$ need not be a full matrix ring. However, the results proved so far do show that an $n$-genus ring satisfies many of the properties of an $n \times n$ matrix ring. As we next prove, it has the useful extra property that it frequently descends to subrings. This implies that the subring will have a decomposition (5.6.1) and will, in turn, make it considerably easier to determine whether that subring is a full matrix ring.

5.7. Proposition. Suppose that $T \subseteq M$ are right Noetherian rings such that $T$ and $M$ have a common ideal $K$. Assume that

(a) $T=\bigoplus_{i=1}^{n} P_{i}$, for some right ideals $P_{i}$ of $T$, and

(b) for all $i$ and $j, P_{i} \otimes_{T} M$ is in the genus of $P_{j} \otimes_{T} M$ as $M$-modules and $\bar{P}_{i}=\left(P_{i}+K\right) / K$ is in the genus of $\bar{P}_{j}$ as modules over $\bar{T}=T / K$.

Then $P_{i}$ is in the genus of $P_{j}$, for all $i$ and $j$.

Proof. The following observations will prove useful. If $L$ is an ideal of $T$, then $L=\bigoplus_{i=1}^{n} P_{i} L$. Thus, $P_{j} \cap L=P_{j} L$ and $\left(P_{j}+L\right) / L \cong P_{j} / P_{j} L$ for $1 \leqslant j \leqslant n$. Also, as $P_{i}$ is a summand of $T$, note that $P_{i} \otimes_{T} M \cong P_{i} M$ for each $i$.

Fix $1 \leqslant i, j \leqslant n$. In order to prove the proposition, it suffices to show that $\rho\left(P_{i}, I\right)=\rho\left(P_{j}, I\right)$ for all prime ideals $I$ of $T$. Assume, first, that $I \supseteq K$. Then, $P_{j} / P_{j} K \cong \bar{P}_{j}$ is in the genus of $\bar{P}_{i} \cong P_{i} / P_{i} K$ as $\bar{T}$-modules. Thus, $\rho\left(P_{i}, I\right)=$ $\rho\left(P_{i} / P_{i} K, I / K\right)=\rho\left(P_{j}, I\right)$ for all $i$ and $j$.

Suppose, instead, that $I \supsetneqq K$. By [15, Example 3.6.3(iii)], there exists a prime ideal $J$ of $M$ such that $J \cap T=I$. Clearly, $K \nsubseteq J$ and so $(K+J) / J$ is a non-zero ideal both of $M / J$ and of $T^{\prime}=(T+J) / J \cong T / I$. In particular, the ring of fractions $Q(T / I) \cong Q\left(T^{\prime}\right)=Q(M / J)=K \cdot Q(M / J)$. By the first paragraph, $P_{i} / P_{i} I \cong$ $P_{i} /\left(P_{i} \cap J\right) \cong\left(P_{i}+J\right) / J$. Therefore,

$$
\begin{aligned}
\widetilde{P}_{i} & =P_{i} / P_{i} I \otimes_{T / I} Q(T / I) \\
& \cong\left(\left(P_{i}+J\right) / J\right) \otimes_{T^{\prime}} Q(M / J) \\
& \cong\left(\left(P_{i} K+J\right) / J\right) \otimes_{T} Q(M / J) \\
& \cong\left(\left(P_{i} M+J\right) / J\right) \otimes_{M / J} Q(M / J) \\
& \cong\left(P_{i} M / P_{i} J\right) \otimes_{M / J} Q(M / J) .
\end{aligned}
$$

By hypothesis, $P_{i} M$ is in the genus of $P_{j} M$. Thus, the displayed equation implies that $\widetilde{P}_{i} \cong \widetilde{P}_{j}$. Equivalently, $\rho\left(P_{i}, I\right)=\rho\left(P_{j}, I\right)$, as required. 
We are now ready to show that, in many circumstances, the property of being an $n$-genus ring descends to subrings.

5.8. TheOREM. Let $T \subseteq M$ be right Noetherian rings, with a common ideal, say $J \subset T \subseteq M$, such that $\bar{T}=T / J$ and $\bar{M}=M / J$ are semilocal rings.

(i) If $M$ and $\bar{T}$ are $n$-genus rings, then $T$ is an $n$-genus ring.

(ii) If, further, $M$ is an $n \times n$ matrix ring, then there exists a ring $R$ such that $M=M_{n}(R)$ and $T$ is a tiled subring of $M_{n}(R)$.

Proof. (i) Throughout the proof, given a subset $X \subseteq M$, we will write $\bar{X}$ to denote the image of $X$ in $\bar{M}=M / J$. Let $M=\bigoplus Q_{i}$ be the given decomposition of $M$ as an $n$-genus ring. Then we may write $Q_{j}=E_{j} M$, for some orthogonal idempotents $E_{j}$ with $\sum E_{i}=1$. Set $E_{j}=\bar{E}_{j}$. Since $\bar{Q}_{j} \cong Q_{j} / Q_{j} J$, clearly all $\bar{Q}_{j}$ are in the same genus as $\bar{M}$-modules. Thus, Lemma 5.2(i) implies that $e_{i} \bar{M}=\bar{Q}_{i} \cong e_{j} \bar{M}$ for all $i$ and $j$. In other words, the idempotents $e_{j}$ are the diagonal elements $e_{j}=e_{j j}$ of a complete set of matrix units $\left\{e_{j l}\right\}$ in $\bar{M}$. Now, by hypothesis and Lemma 5.2(ii), $\bar{T}$ is an $n \times n$ matrix ring. Consequently, $\bar{T}$ (and hence $\bar{M}$ ) also contains a set of $n \times n$ matrix units $\left\{d_{i j}\right\}$. Therefore, $\bar{M} \cong\left(d_{j j} \bar{M}\right)^{(n)} \cong\left(e_{j j} \bar{M}\right)^{(n)}$, for each $j$. Since $\bar{M}$ is a semilocal ring, Lemma 5.2(i) implies that $d_{j j} \bar{M} \cong e_{j j} \bar{M}$ for all $j$.

In this situation [12, Corollary 1.12] implies that the orthogonal idempotents $d_{i i}$ can be lifted to orthogonal idempotents $D_{i} \in M$ such that $\sum_{i} D_{i}=1$ and $D_{i} M \cong E_{i i} M$ for all $i$. Since $d_{i i}=\bar{D}_{i}$, each $D_{i} \in T$. Thus, if $P_{i}=D_{i} T$, then $T=\bigoplus_{j=1}^{n} P_{j}$. The fact that the $P_{j}$ are all in the same genus now follows from Proposition 5.7.

(ii) In this case, we may assume that $Q_{j}=E_{j} M$, for $1 \leqslant j \leqslant n$, where the $E_{j}=E_{j j}$ are the diagonal elements in the given set of matrix units $\left\{E_{i j}\right\}$ for $M$. The comments of the last paragraph now imply that $D_{j} M \cong E_{j} M \cong E_{i} M \cong D_{i} M$, for all $i$ and $j$. Thus, the $D_{j}$ are actually the diagonal elements $D_{j}=D_{j j}$ of a complete set of matrix units $\left\{D_{j l}\right\}$ of $M$. View the elements $D_{j l}$ as the standard matrix units in a new matrix representation $M=M_{n}(R)$ of $M$. If $R_{i j}$ denotes the set of $(i, j)$ th entries of the matrices in $D_{i i} T D_{j j}$, then this implies that $T$ equals the tiled subring $\left(R_{i j}\right)$ of $M_{n}(R)$.

5.9. Corollary. Let $T \subset M=M_{n}(R)$ be prime, right Noetherian rings of Krull dimension 1 that satisfy the hypotheses of Question 1.1. Then

$$
T \cong \operatorname{End}_{S}\left(S^{(n-1)} \oplus L\right)
$$

for some ring $S$ Morita equivalent to $T$ and right ideal $L$ of $S$. Moreover, there exists a new matrix presentation $M_{n}(R)=M_{n}(\widetilde{R})$ such that $T$ is a tiled subring of $M_{n}(\widetilde{R})$.

Proof. If $K=0$, the result is trivial. Otherwise, if $J=M_{n}(K)$, then $T / J$ and $M / J$ are Artinian and so the hypotheses of Theorem 5.8 are satisfied. Now, apply Theorems 5.8 and 5.6.

Remark. One can substantially strengthen this last result. Assume that $T \subseteq M$ satisfy the hypotheses of Theorem 5.8(i) and that $\operatorname{Kdim}(T)=\operatorname{Kdim}(M)=1$. Then, the decomposition given by Theorem 5.6 actually holds simultaneously for $T$ and 
$M$. More precisely: there exist rings $S \subset \tilde{S}$ and a right $S$-module $L$ in the genus of $S$ such that $T \cong \operatorname{End}_{S}\left(S^{(n-1)} \oplus L\right)$ and $M \cong \operatorname{End}_{\tilde{S}}\left(\tilde{S}^{(n-1)} \oplus L \tilde{S}\right)$. Since this result will not be needed later, the details will be omitted.

Corollary 5.9 shows that the ring $T$ defined therein is always close to being an $n \times n$ matrix ring. Moreover, it enables us to give a computationally useful criterion for determining whether or not $T$ actually is such a matrix ring. The critical concept is the genus class group of $S$ (defined below), while the criterion itself is given in Lemma 5.11 .

5.10. Definition. Let $S$ be a right Noetherian ring with $\mathrm{Kdim} S=1$. Two finitely generated, projective right $S$-modules $P$ and $Q$ are stably isomorphic if $P \oplus S^{(r)} \cong Q \oplus S^{(r)}$ for some integer $r$. The genus class group $G(S)$ of $S$ is then defined to be the set of all stable isomorphism classes $[P]$ of $S$-modules $P$ in the genus of $S$. Addition is defined by $[P]+[Q]=[X]$, where $X$ is any $S$-module such that $S \oplus X \cong P \oplus Q$. Note that, by Proposition 5.5(ii), such a module $X$ exists and $[X]$ is uniquely defined. Also, $[S]=0$. Finally, Proposition 5.5 implies that inverses exist. Indeed, if $P \oplus Y \cong S^{(m)}$, then $Y$ is in the genus of $S^{(m-1)}$ and Proposition 5.5(i) implies that $Y \cong S^{(m-2)} \oplus Q$, for some $Q$. Finally, Proposition 5.5(iii) implies that $P \oplus Q \cong S \oplus S$ and so $[Q]=-[P]$ in $G(S)$. When $S$ is commutative, $G(S)$ coincides with the Picard group $\operatorname{pic}(S)$.

5.11. Lemma. Let $T=\operatorname{End}_{S}\left(S^{(n-1)} \oplus L\right)$, where $S$ is a right Noetherian ring of Krull dimension 1 and $L$ is a module in the genus of $S$. Suppose that $n=r t$ for some positive integers $r$ and $t$. Then the following statements are equivalent:

(i) $T$ is an $r \times r$ matrix ring;

(ii) there exists a right $S$-module $X$ such that $S^{(n-1)} \oplus L \cong X^{(r)}$;

(iii) the element $[L]$ of the genus class group $G(S)$ has an rth root.

Proof. (i) $\Leftrightarrow$ (ii). This follows from Lemma 2.1.

(ii) $\Rightarrow$ (iii). To avoid trivialities, assume that $r>1$. Given a prime ideal $I$ of $S$, then $r \rho(X, I)=n \rho(S, I)$ and hence $\rho(X, I)=t \rho(S, I)$ and $X$ is in the genus of $S^{(t)}$. Thus, Proposition 5.5(i) implies that $X \cong S^{(t-1)} \oplus Y$, for some module $Y$ in the genus of $S$. In particular, $L \oplus S^{(n-1)} \cong Y^{(r)} \oplus S^{(n-r)}$ and then Proposition 5.5(iii) yields $L \oplus S^{(r-1)} \cong Y^{(r)}$. Thus, $[L]=r[Y]$ in the genus class group $G(S)$.

(iii) $\Rightarrow$ (ii). Suppose that $r[Y]=[L]$. Then $Y^{(r)} \cong L \oplus S^{(r-1)}$, by the definition of addition in $G(S)$. Thus, $L \oplus S^{(n-1)}=L \oplus S^{(r t-1)} \cong Y^{(r)} \oplus S^{(t r-r)} \cong X^{(r)}$, where $X=$ $Y \oplus S^{(t-1)}$.

We can now reap the benefits of the theory developed in this section to find counter-examples, notably to some of the questions raised in $\S 4$. Indeed, Lemma 5.11 and Corollary 5.9 indicate where one should look: find a ring $S$ with a suitable genus class group (or Picard group) and consider $T=\operatorname{End}_{S}\left(S^{(n-1)} \oplus L\right)$. The next result provides some appropriate choices for $S$. We write $X^{\times}$for the group of units of a ring $X$. 
5.12. Lemma. Let $R=k[x]$ be the polynomial ring over a field $k$.

(i) If $S=k\left[x^{2}, x^{3}\right]$ then $\operatorname{pic}(S) \cong(k,+)$, the additive group of $k$.

(ii) If $S=k+x(x-1) k[x]$, then $\operatorname{pic}(S) \cong k^{\times}$.

Proof. Let c denote the conductor, $\mathbf{c}=\operatorname{Ann}_{S}(R / S)$, and use the well-known formula $\operatorname{pic}(S) \cong(R / \mathbf{c})^{\times} /\left\{(S / \mathbf{c})^{\times} \cdot \operatorname{im}\left(R^{\times}\right)\right\}$, where $\operatorname{im}(R)$ denotes the natural image of $R^{\times}$in $(R / \mathbf{c})^{\times}$. (This formula follows, for example, from the MayerVietoris sequence in $[\mathbf{1 8}$, p. 170].)

All our examples will be constructed in the following manner and, as we show, they automatically satisfy all the basic hypotheses considered in this section.

5.13. ExAmple. Let $S$ be any Noetherian, commutative integral domain of Krull dimension 1 such that the integral closure $R$ of $S$ in $Q(S)$ is a principal ideal domain. Assume that there exists an invertible, non-principal fractional ideal $L$ of $S$. Then, $L R=x R$, for some $x$ and, replacing $L$ by $x^{-1} L$, we may assume that $L R=R$. Note that this implies that $L^{-1} R=R$ and that $L \supseteq \mathrm{c}=\mathrm{Ann}_{S}(R / S)$. Now, let $T$ be the ring

$$
T=\left(\begin{array}{lllll}
S & S & \cdots & S & L^{-1} \\
S & S & \cdots & S & L^{-1} \\
\vdots & \vdots & \ddots & \vdots & \vdots \\
S & S & \cdots & S & L^{-1} \\
L & L & \cdots & L & S
\end{array}\right)_{n \times n} \cong \operatorname{End}_{S}\left(S^{(n-1)} \oplus L\right)
$$

We claim that:

(a) $T$ is a tiled subring of $M_{n}(R)$ such that $T$ and $R$ are prime Noetherian rings of Krull dimension 1; moreover, $T$ is an n-genus ring;

(b) $L$ is in the genus of $S$;

(c) $T \supset J=M_{n}(\mathbf{c})$ and $T / J \cong M_{n}(S / \mathbf{c})$; thus, the hypotheses of Theorem 5.8 are satisfied;

(d) every proper factor ring $\bar{T}$ of $T$ is an $n \times n$ matrix ring.

Part (b) follows from the fact that every projective, fractional ideal of a commutative domain $S$ is in the genus of $S$. Part (a) now follows from the construction of $L$, combined with Proposition 5.4. Since $L R=L^{-1} R=R$, clearly $L \mathbf{c}=L^{-1} \mathbf{c}=\mathbf{c}$ and $T \supset J=M_{n}(\mathbf{c})$. Note that $T / J \cong \operatorname{End}_{S}\left((S / \mathbf{c})^{(n-1)} \oplus L / L \mathbf{c}\right)$. Since $L$ is in the genus of $S$ and $S / \mathbf{c}$ is Artinian, $L / L \mathbf{c} \cong S / \mathbf{c}$. Thus, $T / J \cong M_{n}(S / \mathbf{c})$ and (c) holds. Finally, since $\bar{T}$ is Artinian, part (d) follows from part (a) and Lemma 5.2 .

The only remaining question about the structure of $T$ is whether $T$ is itself an $n \times n$ matrix ring. By Lemma 5.11, this holds if and only if $[L]$ has an $n$th root in $G(S)=\operatorname{pic}(S)$. Thus, using this procedure for the rings defined by Lemma 5.12 gives:

5.14. Proposition. Let $S=k\left[x^{2}, x^{3}\right] \subseteq R=k[x]$, where $k$ is a field of characteristic $p \neq 0$. By Lemma 5.12, pick a non-trivial element $[L] \in \operatorname{pic}(S)$ such that $L R=R$ (an explicit example is $\left.L=x^{2} k[x]+(1-x) k\right)$. For any such $L$, let $T \cong \operatorname{End}_{S}\left(S^{(n-1)} \oplus L\right)$ be defined by (5.13.1).

(i) The ring $T$ is an $n \times n$ matrix ring if and only if $(n, p)=1$. 
(ii) More generally, let $r$ be any positive integer. Then, $T$ is an $r \times r$ matrix ring if and only if $(r, p)=1$ and $r$ divides $n$.

Proof. Note that, as $T$ has a simple Artinian ring of fractions $M_{n}(k(x))$, if $T$ is an $r \times r$ matrix ring, then $r \mid n$. Lemma 5.12 shows that $\operatorname{pic}(S) \cong(k,+)$ and hence that $[L]$ has an $r$ th root in $\operatorname{pic}(S)$ if and only if $(r, p)=1$. The result now follows from Lemma 5.11 .

5.15. Proposition. Let $R=\mathbb{Q}[x] \supseteq S=\mathbb{Q}+x(x-1) \mathbb{Q}[x]$. Then there exists an invertible $S$-module $L$ such that, for all $n$ and all $r>1$, the ring $T \cong \operatorname{End}_{S}\left(S^{(n-1)} \oplus\right.$ $L)$ defined by (5.13.1) is not an $r \times r$ matrix ring.

Proof. In this case $\operatorname{pic}(S) \cong \mathbb{Q}^{\times}$. Therefore, there exists $L$ such that $[L]$ does not have an $r$ th root for any $r>1$. (Explicitly, one could take $L=$ $x(x-1) \mathbb{Q}[x]+(x+a(x-1)) \mathbb{Q}$, where $a$ is any non-zero, square-free integer.) Thus, Lemma 5.11 implies that, for this choice of $L$, the ring $T=$ $\operatorname{End}_{S}\left(S^{(n-1)} \oplus L\right)$ is never an $r \times r$ matrix ring.

5.16. Corollary. Let $r>1$ be an integer. Then there exist a ring $R^{\prime}$ and a tiled subring $T=\left\{T_{i j}^{\prime}\right\} \subseteq M_{r}\left(R^{\prime}\right)$ with the following properties:

(i) there exists an ideal $J$ of $R^{\prime}$ such that $R^{\prime} / J$ is an $r \times r$ matrix ring;

(ii) every tile $T_{i j}^{\prime} \supseteq \mathrm{J}$;

(iii) for every diagonal tile, $T_{i i}^{\prime} / J$ is a full $r \times r$ matrix ring;

(iv) $T$ is not an $r \times r$ matrix ring.

Proof. Take $T$ to be the ring defined by Proposition 5.15, with $n=r^{2}$. Then $T$ is a tiled subring of the $r^{2} \times r^{2}$ matrix ring $M_{r^{2}}(R)$, where $R=k[x]$. For the present example we view $T$, in block form, as a tiled subring of the $r \times r$ matrix ring $M_{r}\left(R^{\prime}\right)$ where $R^{\prime}=M_{r}(R)$. For example, if $r=2$, then

$$
T=\left(\begin{array}{ll}
\left(\begin{array}{ll}
S & S \\
S & S
\end{array}\right) & \left(\begin{array}{cc}
S & L^{-1} \\
S & L^{-1}
\end{array}\right) \\
\left(\begin{array}{ll}
S & S \\
L & L
\end{array}\right) & \left(\begin{array}{cc}
S & L^{-1} \\
L & S
\end{array}\right)
\end{array}\right) .
$$

Thus assertions (i)-(iii) follow immediately from the discussion in (5.13), while (iv) follows from Proposition 5.15.

The significance of Corollary 5.16 is that it shows that hypothesis (ii) in Theorem 4.5 cannot be replaced by the simpler hypothesis 'each $R_{i i} / K$ is an $n \times n$ matrix ring'. Consequently, hypothesis (4.2.1) of Theorem 4.2 also cannot be deleted.

We conclude this section with two results that formalise the intuitive idea that if a ring $T$ satisfies something like the hypotheses of Question 1.1, then 'almost always' $T$ is a full matrix ring. In the first example, we consider the case of tiled subrings of classical orders: 
5.17. TheOREM. Let $R$ be a non-commutative integral domain that is a module-finite algebra over its centre $C$, and suppose that all proper residue rings of $C$ are finite. Let $\Delta$ be the quotient division ring of $R$, and let $n^{2}$ be the dimension of $\Delta$ over its centre.

(i) The 'exceptional' set $\mathscr{E}$ of maximal ideals $N$ such that $R / N$ is not an $n \times n$ matrix ring is finite.

(ii) Let $A=\left(R_{i j}\right)$ be any tiled subring of $M_{n}(R)$ whose diagonal tiles equal $R$ and whose off-diagonal tiles $R_{i j}(i \neq j)$ are ideals of $R$ not contained in any of the maximal ideals in $\mathscr{E}$. Then $A$ is an $n \times n$ matrix ring.

Proof. (i) In the notation of, for example, [15, Chapter 13], $R$ has PI degree $n$, written PI- $\operatorname{deg}(R)=n$. Note that, by hypothesis, if $N$ is a maximal ideal of $R$ then $R / N$ is finite and therefore, by Wedderburn's Theorem on finite division rings, it is a full matrix ring $M_{r}(F)$ over its centre $F$. Here, $r=\mathrm{PI}-\operatorname{deg}(R / N)$. Thus part (i) asserts that PI-deg $(R / N)=\mathrm{PI}-\operatorname{deg}(R)$ for all but finitely many maximal ideals $N$ of $R$. Let $g$ be any central polynomial for $R$ and $I$ the ideal of $R$ generated by $g(R)$. Since $g(R) \neq 0$, the ring $R / I$ is finite and only finitely many maximal ideals of $R$ can contain $I$. For any other maximal ideal $N$ of $R$, [15, Lemma 13.7.2] implies that PI-deg $(R / N)=\operatorname{PI}-\operatorname{deg}(R)$.

(ii) Let $K=\bigcap_{i j} R_{i j}$. By Theorem 4.5, it suffices to show that $R / K$ is an $n \times n$ matrix ring, and for this it suffices to show that $R / K$, considered as a left $R / K$-module, has an $n$th root; that is, $R / K$ is isomorphic to the direct sum of $n$ mutually isomorphic modules. Orthogonal idempotents in any ring can be lifted modulo nil ideals, and generate isomorphic left ideals after the lifting if they did so before the lifting [10, Proposition 1, p. 53]. Therefore it suffices to show that $R / K$, modulo its radical, has an $n$th root. Thus, we can suppose that the finite ring $R / K$ is semisimple, and hence a direct sum of simple rings. By hypothesis, these simple rings are all $n \times n$ matrix rings. Therefore, $R / K$ is also an $n \times n$ matrix ring.

5.18. Proposition. Keep the hypotheses of Theorem 5.8(ii) and suppose that $\mathbf{K}_{1}(M / J)$ has finite exponent $f$, where $n$ is prime to $f$. Then $T$ is an $n \times n$ matrix ring.

Remark. Once again, this result applies frequently. For example, let $R$ be a classical order of number-theoretic origin and $\bar{R}=R / K$ any proper factor ring of $R$. Then, $\bar{R}$ is finite and so certainly has a finite Whitehead group, say of exponent $f$. Now, for any $n$ coprime to $f$, let $T$ be any subring of $M=M_{n}(R)$ such that $J=M_{n}(K) \subset T$ and $T / J$ is an $n \times n$ matrix ring. Then, [18, Proposition 35, p. 109] implies that $\mathbf{K}_{1}(M / J) \cong \mathbf{K}_{1}(\bar{R})$ and so the proposition implies that $T$ is an $n \times n$ matrix ring.

Proof. Set $\bar{M}=M / J$ and $\bar{T}=T / J$ and note that $T$ can be defined in terms of the pullback:

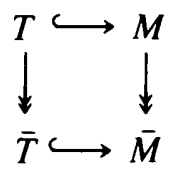


Thus, by [18, Proposition 63, p. 162] one obtains the exact sequence

$$
\mathbf{K}_{1}(\bar{M}) \stackrel{\phi}{\longrightarrow} \mathbf{K}_{0}(T) \stackrel{\theta}{\longrightarrow} \mathbf{K}_{0}(M) \oplus \mathbf{K}_{0}(\bar{T}) \stackrel{\psi}{\longrightarrow} \mathbf{K}_{0}(\bar{M})
$$

where $\theta([P])=\left[P \otimes_{T} M\right]-[\bar{P}]$. Since $M \cong M_{n}(R)$ and $\bar{T}=M_{n}(B)$ for some rings $R$ and $B$, one finds that $\theta([T])=[M]-[\bar{T}]=n V$, for some $V \in \mathbf{K}_{0}(M) \oplus \mathbf{K}_{0}(\bar{T})$. Since $n V \in \operatorname{Im}(\theta), n \psi(V)=\psi(n V)=0$. But, as $\bar{M}$ is Artinian, $\mathbf{K}_{0}(\bar{M})$ is a free abelian group and so this implies that $\psi(V)=0$; that is, $V=\theta(W)$ for some $W \in \mathbf{K}_{0}(T)$. Thus $\theta([T]-n W)=0$ and so $[T]-n W=\phi(Z)$ for some $Z \in$ $\mathbf{K}_{1}(\bar{M})$.

Since $\mathbf{K}_{1}(\bar{M})$ has exponent coprime to $n, Z=n Y$, for some $Y \in \mathbf{K}_{1}(\bar{M})$ and $[T]=n W+n \phi(Y)=n X$ for some $X \in \mathbf{K}_{0}(T)$. We may write $X=[Q]-m[T]$, for some projective $T$-module $Q$. Thus, for some integer $s, T \oplus T^{(m n)} \oplus T^{(s)} \cong$ $Q^{(n)} \oplus T^{(s)}$. This implies that $\rho(Q, I)=(m+1 / n) \rho(T, I)$, for each prime ideal $I$ of $T$. As usual, use Proposition 5.7 to write $T=\bigoplus_{j=1}^{n} P_{j}$, where each $P_{j}$ is in the same genus. Thus, $\rho(Q, I)=(m n+1) \rho\left(P_{1}, I\right)$ for all prime ideals $I$ of $T$. Thus, Proposition 5.5(ii) implies that $Q \cong Q_{1} \oplus T^{(m)}$ for some module $Q_{1}$. Therefore, $T^{(t)} \oplus T \approx Q_{1}^{(n)} \oplus T^{(t)}$, for $t=m n+s$. Finally, Proposition 5.5(iii) implies that $T \cong Q_{1}^{(n)}$, as required.

\section{Genus class groups and counter-examples}

In this section we determine when certain rings of the form $T_{n}=R+M_{n}(K)$, where $K$ is an ideal of $R$, are full $n \times n$ matrix rings. The answer is subtle, and depends on a detailed analysis of the genus class groups of such rings. In addition to proving Theorem 1.6, this analysis shows the following.

- The hypothesis $\operatorname{det}(\bar{\alpha})=1$ cannot be deleted from Theorem 3.3 or Corollary 3.4 .

- The hypothesis about roots of -1 in Theorem 4.2 (ii) cannot be deleted. Consequently, the hypothesis that $\operatorname{det} \omega=1$ in part (iii) of that theorem also cannot be deleted.

- If one is working with $n \times n$ matrices for $n \equiv 2(\bmod 4)$, then the hypothesis about roots of -1 in Theorem 4.8 (i) cannot be deleted.

- Suppose that $n=2$. Then, in Theorems 4.8(i) and 4.2(ii), one also cannot replace the hypothesis that $V-1 \in Z(R)$ by the simpler assertion that there exists $x \in R$ such that $x^{2}=-1$.

We fix the following notation throughout the section, for consistency with the notation of Example 4.7(iii) and Theorem 4.8.

6.1. Notation. We use $S^{\times}$to denote the multiplicative group of units in a ring $S$. Let $R$ be any semiprime ring, with zero socle, that is a module-finite algebra over a central, Noetherian ring $C$ of Krull dimension 1 . (We shall ultimately take $R$ to be either $\mathbb{H}$ or the standard maximal order $\mathbf{H}$ that contains it.) We assume that there is a surjective $C$-algebra homomorphism $\psi: R \rightarrow M_{2}(F)$ for some field 
$F$, and define $\psi_{2}: M_{2}(R) \rightarrow M_{4}(F)$ by letting $\psi$ act coordinatewise. Let $K=\operatorname{ker} \psi$, and, as in Examples 4.7(iii), set

$$
T=R+M_{2}(K)=\left\{\left(\begin{array}{rr}
r+k_{1} & k_{2} \\
k_{3} & r+k_{4}
\end{array}\right): r \in R \text { and } k_{j} \in K\right\},
$$

a subring of $M_{2}(R)$. Note that $\bar{T}=\psi_{2}(T)$ consists of all matrices $\alpha \in M_{4}(F)$ of the form:

$$
\alpha=\left(\begin{array}{cccc}
a & b & 0 & 0 \\
c & d & 0 & 0 \\
0 & 0 & a & b \\
0 & 0 & c & d
\end{array}\right) \quad \text { for } a, b, c, d \in F
$$

Thus $\bar{T}$ is a non-tiled subring of $M_{4}(F)$, and $\bar{T}$ is isomorphic to $M_{2}(F)$. Indeed, as was observed in Examples $4.7, \bar{T}$ is permutation-isomorphic to a $2 \times 2$ matrix subring of $M_{4}(F)$, by means of a permutation matrix $\omega$ of determinant -1 . If $Z(R)$ contains a square root of -1 , it follows from Theorem 4.2 that $T$ is isomorphic to a $2 \times 2$ matrix ring.

Throughout this section, we will use the concept of genus defined in (5.1). As was remarked there, this definition coincides with the classical one, for finitely generated projective modules over classical orders.

6.2. Remark. To motivate the computations that follow, we first show that $T$ is a 2-genus ring; that is $T$ has a decomposition $T=U \oplus W$ where $U$ and $W$ are (projective) right $T$-modules in a single genus. By (6.1.1), $M_{2}(K) \subset T \subseteq M_{2}(R)$. Recall that $\bar{T}=T / M_{2}(K)$ is a $2 \times 2$ matrix ring; hence it and (likewise) $M_{2}(R)$ are 2-genus rings. Since all rings involved are Noetherian, and $\bar{T}$ and $M_{2}(R) / M_{2}(K)$ are Artinian, it now follows from Theorem 5.8 that $T$ is a 2-genus ring.

Thus the problem to be solved is to determine whether the summands $U, W$, which are in a single genus, can be replaced by a pair of summands in a single isomorphism class. For then Lemma 2.1 implies that $T$ is a $2 \times 2$ matrix ring. Our final results show that the answer is sometimes yes, sometimes no. We begin with a definition from [8, Notation 3.3] and two lemmas.

For $\gamma \in M_{4}(F)^{\times}$, define $T^{\gamma}=\left\{\alpha \in M_{2}(R): \psi_{2}(\alpha) \in \gamma \bar{T}\right\}$. Observe that $T^{\gamma}$ is a right $T$-module, since $\bar{T}$ is left-multiplied by $\gamma$. It will be convenient to regard $T$ and $T^{\gamma}$ in terms of pullback diagrams. Indeed $T$ and $T^{\gamma}$ are the pullbacks of the first and second cartesian squares, respectively, in the following diagram:

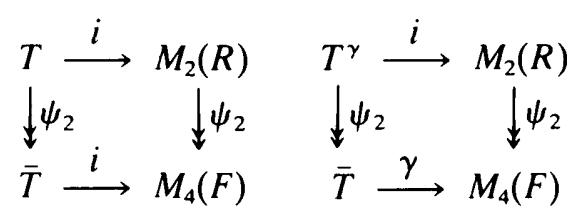

Here, $i$ denotes the inclusion map and $\gamma$ acts by left multiplication. Note that $T=T^{\prime}$, where $I$ is the $4 \times 4$ identity matrix. We shall have several occasions to use the following formula, from [8, Corollary 3.6]:

$$
T^{\alpha} \oplus T^{\beta} \cong T^{\alpha \beta} \oplus T
$$


6.3. Lemma. (i) For any $\gamma \in M_{4}(F)^{\times}$, the module $T^{\gamma}$ is a finitely generated projective right $T$-module in the genus of $T$.

(ii) Let $\omega=(2,3)$ be the $4 \times 4$ permutation matrix such that left multiplication by $\omega$ interchanges rows 2 and 3 of $M_{4}(F)$. Then $T^{\omega} \cong V \oplus V$ for some right $T$-module $V$.

Proof. (i) The module $T^{\gamma}$ is in the genus of $T$ by [8, Theorem 3.4]. To see that $T^{\gamma}$ is projective, take $\alpha=\gamma$ and $\beta=\gamma^{-1}$ in (6.2.2).

(ii) We have

$$
\psi_{2}\left(T^{\omega}\right)=\left\{\left(\begin{array}{llll}
a & b & 0 & 0 \\
0 & 0 & a & b \\
c & d & 0 & 0 \\
0 & 0 & c & d
\end{array}\right): a, b, c, d \in F\right\} \subseteq M_{4}(F)
$$

Let $\left\{f_{i j}\right\}$ denote the standard matrix units of $M_{2}(R)$. Since $\psi(1)$ is the $2 \times 2$ identity matrix, it follows easily from (6.3.1) that $T^{\omega}=f_{11} T^{\omega} \oplus f_{22} T^{\omega}$. Moreover, left multiplication by the permutation matrix $f_{12}+f_{21} \in M_{2}(R)$ provides an isomorphism $f_{11} T^{\omega} \cong f_{22} T^{\omega}$.

Let $\mathscr{T}=\left\{\right.$ isomorphism classes of $\left.T^{\gamma}: \gamma \in M_{4}(R)^{\times}\right\}$. The next lemma provides an alternative description of $\mathscr{T}$. Define $\mathcal{N}=\left\{\operatorname{det} \psi_{2}(U): U \in M_{2}(R)^{\times}\right\}$and $F^{2}=$ $\left\{b^{2}: b \in F^{\times}\right\}$.

6.4. Lemma. (i) The set $\mathcal{T}$ is the set of isomorphism classes of right $T$-modules $P$, in the genus of $T$, for which $P \otimes_{T} M_{2}(R) \cong M_{2}(R)$ as right $M_{2}(R)$-modules.

(ii) The map $T^{\gamma} \mapsto(\operatorname{det} \gamma) \cdot\left(\mathcal{N} \cdot F^{2}\right)$ provides a bijection between $\mathscr{T}$ and the abelian group $F^{\times} /\left(\mathcal{N} \cdot F^{2}\right)$.

(iii) We have $T^{\alpha} \oplus T^{\beta} \cong T^{\gamma} \oplus T^{\delta}$ if and only if $(\operatorname{det} \alpha \beta) \cdot\left(\mathcal{N} \cdot F^{2}\right)=$ $(\operatorname{det} \gamma \delta) \cdot\left(\mathcal{N} \cdot F^{2}\right)$.

Proof. (i) This is a special case of [8, Theorem 3.4].

(ii) The same result from [8] provides a one-to-one correspondence between $\mathscr{T}$ and the collection of double cosets $X=\psi_{2}\left(M_{2}(R)^{\times}\right) \backslash M_{4}(F)^{\times} / \bar{T}^{\times}$given by the correspondence $T^{\gamma} \rightarrow \psi_{2}\left(M_{2}(R)^{\times}\right) \cdot \gamma \cdot \bar{T}^{\times}$. We need to simplify the definition of $X$. Consider the determinant function det: $M_{4}(F)^{\times} \rightarrow F^{\times}$, with kernel $\operatorname{SL}_{4}(F)$. Since $F$ is a field, $\mathrm{SL}_{4}(F)=E(4, F)$, the subgroup generated by the transvections. Moreover, Corollary 3.4(i) implies that $E(4, F) \subseteq \psi_{2}\left(M_{2}(R)^{\times}\right)$. Thus, det induces a bijection between $X$ and $\mathcal{N} \backslash F^{\times} / \operatorname{det}\left(\bar{T}^{\times}\right)=F^{\times} /\left(\mathcal{N} \cdot \operatorname{det}\left(\bar{T}^{\times}\right)\right)$. Finally, (6.1.2) implies that $\operatorname{det}\left(\bar{T}^{\times}\right)=F^{2}$, as required.

(iii) By formula (6.2.2), the statement we are proving is equivalent to the statement

(6.4.1) $\quad T^{\alpha \beta} \oplus T \cong T^{\gamma \delta} \oplus T \Leftrightarrow(\operatorname{det} \alpha \beta) \cdot\left(\mathcal{N} \cdot F^{2}\right)=(\operatorname{det} \gamma \delta) \cdot\left(\mathcal{N} \cdot F^{2}\right)$.

The implication $(\Leftarrow)$ therefore follows from statement (ii). The implication $\Leftrightarrow$ ) follows similarly, if we can cancel $T$ from the isomorphism in (6.4.1). Since $T=U \oplus W$ is a 2-genus ring (Remark 6.2), this follows from Proposition 5.5(iii), with $X=U$. 


\subsection{Theorem. Let $T$ and $R$ be defined as in Notation 6.1.}

(i) If $-1 \in \mathcal{N} \cdot F^{2}$, then $T \cong M_{2}(S)$ for some ring $S$.

(ii) Assume that every $M_{2}(R)$-module in the genus of $M_{2}(R)$ is isomorphic to $M_{2}(R)$. Then $T \cong M_{2}(S)$ for some ring $S$ if and only if $-1 \in \mathcal{N} \cdot F^{2}$.

Proof. Recall that $T$ is a $2 \times 2$ matrix ring if and only if $T_{T} \cong X \oplus X$, for some $T$-module $X$. Also, in the notation of Lemma 6.3(ii), $T^{\omega} \cong V \oplus V$ and det $\omega=-1$.

(i) Suppose that $T$ is not a $2 \times 2$ matrix ring. Then, by the previous paragraph, $T \not T^{\omega}$. Since $T=T^{l}$, Lemma 6.4(ii) implies that $-1=\operatorname{det} \omega \notin \mathcal{N} \cdot F^{2}$.

(ii) Conversely, suppose that $T \cong X \oplus X$ and that every module in the genus of $M_{2}(R)$ is isomorphic to $M_{2}(R)$. By Lemma 6.3(i), $T^{\omega}$ is in the genus of $T$ and hence $X$ is in the genus of $V$. Thus, $Y=V \oplus X$ is in the genus of $T$. Consequently, $Y \otimes_{T} M_{2}(R)$ is in the genus of $M_{2}(R)$ which, by hypothesis, implies that $Y \otimes_{T} M_{2}(R) \cong M_{2}(R)$. Thus, by Lemma 6.4(i), $Y \cong T^{\gamma}$ for some $\gamma$. By construction, $Y \oplus Y \cong(X \oplus X) \oplus(V \oplus V)$; equivalently, $T^{\gamma} \oplus T^{\gamma} \cong T \oplus T^{\omega}$. Since $T=T^{\prime}$, Lemma 6.4(iii) shows that $(\operatorname{det} \omega)\left(\mathcal{N} \cdot F^{2}\right)=(\operatorname{det} \gamma)^{2}\left(\mathcal{N} \cdot F^{2}\right)$. Since $\operatorname{det} \omega=-1$ and $(\operatorname{det} \gamma)^{2} \in F^{2}$, this implies that $-1 \in \mathcal{N} \cdot F^{2}$, as required.

With our machinery established, we now return to the quaternions. We emphasise that, throughout this section, $\mathbf{H}$ denotes the standard maximal order in the rational quaternions, $\mathbf{H}=\mathbb{Z}[i, j, k]+\mathbb{Z} \cdot\left(\frac{1}{2}(1+i+j+k)\right)$. As noted in Lemma $2.2, \mathbf{H} / p \mathbf{H} \cong M_{2}(\mathbb{Z} / p \mathbb{Z})$. Fix $F=\mathbb{Z} / p \mathbb{Z}$. Two important facts about this pair of rings $\mathbf{H}$ and $F$ are provided by the following lemma.

6.6. Lemma. (i) There is a surjective ring homomorphism $\psi: \mathbf{H} \rightarrow M_{2}(F)$, and every such $\psi$ satisfies $\operatorname{det} \psi(u)=1$ for every $u \in \mathbf{H}^{\times}$.

(ii) Let $\psi_{2}: M_{2}(\mathbf{H}) \rightarrow M_{4}(F)$ be the homomorphism that applies $\psi$ coordinatewise. Then $\operatorname{det} \psi_{2}(U)=1$ for every $U \in M_{2}(\mathbf{H})^{\times}$.

Proof. (i) The existence of $\psi$ follows from Lemma 2.2. Let $h=a_{0}+a_{1} i+a_{2} j+a_{3} k \in \mathbf{H}$. The norm function $v(h)=\sum_{i}\left(a_{i}\right)^{2}$ is a multiplicative function from $\mathbf{H}$ to the positive integers, and hence takes units of $\mathbf{H}$ to positive units of $\mathbb{Z}$. So $v\left(\mathbf{H}^{\times}\right)=1$. Thus it suffices to prove that

$$
\operatorname{det}(\psi(h))=\psi(v(h)) \text { for all } h \in \mathbf{H} .
$$

Since $F$ has characteristic $p$, localization at $p$ extends $\psi$ to a ring homomorphism of $\mathbf{H}_{p}$ onto $M_{2}(F)$. It will be easier to prove (6.6.1) for all $h \in \mathbf{H}_{p}$. (Note that the norm function $v$ now maps $\mathbf{H}_{p}$ to $\mathbb{Z}_{p}$ ).

Since $p$ is odd, 2 has a reciprocal in $\mathbf{H}_{p}$, and so $\mathbf{H}_{p}=\mathbb{Z}_{p}[i, j, k]$; that is, we no longer need the generator $\frac{1}{2}(1+i+j+k)$. Therefore, writing bars for images under $\psi$, we have $\overline{\mathbf{H}}=F[\bar{l}, \bar{\jmath}, \bar{k}] \cong M_{2}(F)$. Thus (6.6.1), after localization at $p$, can be rewritten

$$
\operatorname{det} \bar{h}=\sum_{i} \bar{a}_{i}^{2} \quad \text { where } \bar{h}=\bar{a}_{0}+\bar{a}_{1} \bar{l}+\bar{a}_{2} \bar{\jmath}+\bar{a}_{3} \bar{k} \quad\left(\text { for } \bar{a}_{i} \in F\right) .
$$

Note that $\mathbf{H}$ itself is now out of the picture. We are working in the simple $F$-algebra $\overline{\mathbf{H}}=M_{2}(F)$. It suffices to prove (6.6.2) in the larger algebra obtained by 
replacing $F$ by a larger field. So we can suppose that $F$ contains a square root $x$ of -1 . Define $i^{\prime}, j^{\prime}, k^{\prime}$ by

$$
i^{\prime}=\left(\begin{array}{cc}
x & 0 \\
0 & -x
\end{array}\right), \quad j^{\prime}=\left(\begin{array}{cc}
0 & 1 \\
-1 & 0
\end{array}\right), \quad k^{\prime}=\left(\begin{array}{ll}
0 & x \\
x & 0
\end{array}\right) .
$$

Then $\overline{\mathbf{H}}=F\left[i^{\prime}, j^{\prime}, k^{\prime}\right]$ (by counting dimensions), and the correspondence $\bar{l} \rightarrow i^{\prime}$, $\bar{\jmath} \rightarrow j^{\prime}, \bar{k} \rightarrow k^{\prime}$ define an $F$-algebra automorphism of $\overline{\mathbf{H}}$ which, by the SkolemNoether Theorem, is inner. Since inner automorphisms leave determinants unchanged, it suffices to prove $(6.6 .2)$ with $\bar{l}, \bar{j}, \bar{k}$ replaced by the matrices in (6.6.3). It is now routine to verify that

$$
\bar{h}=\left(\begin{array}{cc}
\bar{a}_{0}+\bar{a}_{1} x & \bar{a}_{2}+\bar{a}_{3} x \\
-\bar{a}_{2}+\bar{a}_{3} x & \bar{a}_{0}-\bar{a}_{1} x
\end{array}\right)
$$

and hence (6.6.2) holds.

(ii) Let $U \in M_{2}(\mathbf{H})^{\times}$. By [6, $\$ 91$, Lemma 2], $\mathbf{H}$ is a Euclidean domain. As is proved in [21, Theorem 10.1], this implies that each matrix has a Smith normal form. In other words, $U=P V Q$, where $V=\operatorname{diag}(a, b)$ is a diagonal matrix and the matrices $P$ and $Q$ are products of elementary matrices. Indeed, as is true of a commutative Euclidean domain, we can even take $P$ and $Q$ to be products of transvections (the details are given in the next paragraph.) Since $U \in M_{2}(\mathbf{H})^{\times}$, this implies that $a, b \in \mathbf{H}^{\times}$. Applying $\psi_{2}$ to $U=P V Q$, taking determinants, and applying (i) now completes the proof of (ii).

Wedderburn showed that every matrix $A$ over any (non-commutative) Euclidean domain $R$ has the form $A=P V Q$, where $V$ is a diagonal matrix, while $P$ and $Q$ are products of three types of matrices:

(i) transvections,

(ii) matrices obtainable from the identity matrix by interchanging two of its rows, and

(iii) matrices obtainable from an identity matrix by multiplying one of its non-zero entries by a unit of $R$.

In the previous paragraph we used the fact that the factors of Types (ii) and (iii) are unnecessary. To prove this, note that every matrix of Type (ii) equals a product of transvections and a matrix of Type (iii). Moreover, matrices of Type (iii) normalize the set of transvections and therefore can be moved to the inside of the product $P V Q$, where they do not affect the claimed diagonal form of $V$.

We can finally show that $T$ need not be a $2 \times 2$ matrix ring.

6.7. TheOREM. Let $p$ be an odd prime and $T=\mathbf{H}+M_{2}(p \mathbf{H})$, the ring defined in (6.1.1) with $R=\mathbf{H}$ and $F=\mathbb{Z} / p \mathbb{Z}$. Then $T$ is a $2 \times 2$ matrix ring if and only if $p \equiv 1(\bmod 4)$.

Proof. By [6, §91, Lemma 2], $R=\mathbf{H}$ is a Euclidean domain and so the genus of $M_{2}(R)$ consists of a single isomorphism class, so Theorem 6.5 applies. Moreover, Lemma 6.6(ii) shows that $\mathcal{N}=\{1\}$. Therefore, $T$ is a $2 \times 2$ matrix ring if and only if -1 is a square in $F=\mathbb{Z} / p \mathbb{Z}$, that is, if and only if $p \equiv 1(\bmod 4)$.

We now obtain Theorem 1.6 of the introduction. 
6.8. COROLlary. Let $\mathbb{H}=\mathbb{Z}[i, j, k]$, the ring of integer quaternions, and let $p$ be an odd prime number. Set $T(\mathbb{H})=\mathbb{H}+M_{2}(p \mathbb{H})$, where $\mathbb{H}$ is identified with the ring of scalars inside $M_{2}(\mathbb{H})$. Then $T(\mathbb{H}) \cong M_{2}(S)$, for some ring $S$ if and only if $p \equiv 1(\bmod 4)$.

Proof. Write $T(\mathbf{H})$ for the ring $T$ in Theorem 6.7. If $p \equiv 3(\bmod 4)$ then the ring $T(\mathbf{H})$ is not a $2 \times 2$ matrix ring, by Theorem 6.7. Hence, by Lemma 2.1, neither is its subring $T(\mathbb{H})$. Conversely, suppose that $p \equiv 1(\bmod 4)$. Then -1 is a square in $F$ and Theorem $6.5(\mathrm{i})$ implies that $T(\mathbb{H})$ is a $2 \times 2$ matrix ring.

Note that this corollary also shows that, for $n=2$, Theorem 4.8(i) will not hold if one merely assumes that there exists $x \in R$ such that $x^{2}=-1$. Consequently, the same is true of Theorem 4.2(ii). As we show in the next proposition, the same assertion holds for any positive integer $n$ of the form $n \equiv 2$ modulo 4 .

6.9. Lemma. Let $p \equiv 3(\bmod 4)$ be an odd prime, $T=\mathbf{H}+M_{2}(p \mathbf{H})$ the ring defined in Theorem 6.7, and $r$ an odd number. Then the ring $M_{r}(T)$ is not isomorphic to a full $2 \times 2$ matrix ring.

Proof. This is similar to the proof of Theorem 6.5. Suppose that $M_{r}(T) \cong$ $M_{2}(A)$. Then $M_{r}(T) \cong\left(X^{\prime}\right)^{(2)}$ for some right $M_{r}(T)$-module $X^{\prime}$. The familiar Morita equivalence between $M_{r}(T)$ and $T$ takes $M_{r}(T)$ to $T^{(r)}$. Therefore we have $T^{(r)} \cong X^{(2)}$ for some $T$-module $X$. Let $\omega$ be as in Lemma 6.3(ii). Since $T$ is in the genus of $T^{\omega}=V^{(2)}$, this implies that $X$ is in the genus of $V^{(r)}$. Set $Y=V^{(r)} \oplus X$ and note that $Y$ is in the genus of $T^{(r)}$. By Proposition 5.5(i), we may write $Y=T^{(r-1)} \oplus Z$, where $Z$ is in the genus of $T$. Therefore $Z \otimes M_{2}(\mathbf{H})$ is in the genus of the $M_{2}(\mathbf{H})$-module $M_{2}(\mathbf{H})$. Since $\mathbf{H}$ is a principal ideal domain [6], we have $Z \otimes M_{2}(\mathbf{H}) \cong M_{2}(\mathbf{H})$ as $Z \otimes M_{2}(\mathbf{H})$-modules. Thus, by Lemma 6.4(i), $Z \cong T^{\gamma}$ for some $\gamma$. Consequently,

$$
\left(T^{\gamma}\right)^{(2)} \oplus T^{(2 r-2)} \cong Y^{(2)} \cong V^{(2 r)} \oplus X^{(2)} \cong\left(T^{\omega}\right)^{(r)} \oplus T^{(r)} .
$$

Thus, repeated use of the proof of Lemma 6.4(iii) yields

$$
(\operatorname{det} \gamma)^{2}\left(\mathcal{N} \cdot F^{2}\right)=(\operatorname{det} \omega)^{r}\left(\mathcal{N} \cdot F^{2}\right) .
$$

Since $r$ is odd, $(\operatorname{det} \omega)^{r}=-1$. Since $(\operatorname{det} \gamma)^{2} \in F^{2}$, this implies that $-1 \in \mathcal{N} \cdot F^{2}$. By Theorem $6.5, T$ is a $2 \times 2$ matrix ring, which contradicts Theorem 6.7 .

The next result shows that the situation $n \equiv 2(\bmod 4)$, with $V-1 \notin Z(R)$, is a genuine exception in Theorem 4.8(i).

6.10. Proposition. For every positive integer $n \equiv 2(\bmod 4)$ there exists a ring $\Theta=R^{\prime}+M_{n}\left(K^{\prime}\right)$, the $n \times n$ analogue of the ring $T$ in (6.1.1) (thus $R^{\prime} / K^{\prime}$ is an $n \times n$ matrix ring), such that $\Theta$ is not isomorphic to a full $n \times n$ matrix ring. Indeed, $\Theta$ is not even isomorphic to a $2 \times 2$ matrix ring.

Proof. The construction is as follows. Let $p$ be an odd prime congruent to 3 modulo 4 and write $n=2 u$. Set $R^{\prime}=M_{u}(\mathbf{H}) \supset K^{\prime}=M_{u}(p \mathbf{H})$. Thus, $R^{\prime} / K^{\prime} \cong$ $M_{n}(F)$, where $F=\mathbb{Z} / p \mathbb{Z}$. As usual, identify $R^{\prime}$ with the scalar matrices inside $M_{n}\left(R^{\prime}\right)$ and set $\Theta=R^{\prime}+M_{n}\left(K^{\prime}\right)$. 
Consider the usual identification of $M_{2 u}\left(R^{\prime}\right)=M_{2 u}\left(M_{u}(\mathbf{H})\right) \cong M_{2 u^{2}}(\mathbf{H})$. This gives the following identification of $\Theta$ with a subring of $M_{2 u^{2}}(\mathbf{H})$ :

$$
\Theta=\left\{\left(\begin{array}{cccc}
\left(z_{i j}\right) & 0 & \cdots & 0 \\
0 & \left(z_{i j}\right) & \cdots & 0 \\
\vdots & \vdots & \ddots & \vdots \\
0 & 0 & \cdots & \left(z_{i j}\right)
\end{array}\right)_{2 u \times 2 u}:\left(z_{i j}\right) \in R^{\prime}=M_{u}(\mathbf{H})\right\}+M_{2 u^{2}}(p \mathbf{H})
$$

We next want to show that there exists an embedding $\Theta \hookrightarrow M_{u^{2}}(T)$, where $T=\mathbf{H}+M_{2}(p \mathbf{H})$ is the ring defined by Example 6.7. The diagonal embedding $\mathbf{H} \hookrightarrow M_{2}(\mathbf{H})$ induces an embedding $A=M_{u^{2}}(\mathbf{H}) \hookrightarrow M_{u^{2}}\left(M_{2}(\mathbf{H})\right) \cong M_{2 u^{2}}(\mathbf{H})=B$. Now apply the natural isomorphism $\psi: M_{u^{2}}\left(M_{2}(\mathbf{H})\right) \rightarrow M_{2}\left(M_{u^{2}}(\mathbf{H})\right.$ ). (If one identifies both rings with $M_{2 u^{2}}(\mathbf{H})$, then $\psi$ is just conjugation by the matrix $\omega$ in Definition 4.3.) This gives

$$
\psi(A)=\left\{\left(\begin{array}{cc}
\left(r_{i j}\right) & 0 \\
0 & \left(r_{i j}\right)
\end{array}\right):\left(r_{i j}\right) \in M_{u^{2}}(\mathbf{H})\right\} \subset \psi(B)=M_{2 u^{2}}(\mathbf{H}),
$$

as in the proof of Lemma 4.4. Thus, under the same homomorphism:

$$
\psi\left(M_{u^{2}}(T)\right)=\left\{\left(\begin{array}{cc}
\left(r_{i j}\right) & 0 \\
0 & \left(r_{i j}\right)
\end{array}\right):\left(r_{i j}\right) \in M_{u^{2}}(\mathbf{H})\right\}+M_{2 u^{2}}(p \mathbf{H})
$$

The basic observation is that, as the matrix $\left(z_{i j}\right)$ appears an even number of times in (6.10.1), the identifications of $(6.10 .1)$ and (6.10.2) provide an embedding $\Theta \hookrightarrow \psi\left(M_{u^{2}}(T)\right) \cong M_{u^{2}}(T)$.

Since $u$ is odd, Corollary 6.9 implies that $M_{u^{2}}(T) \neq M_{2}(U)$ for any ring $U$. Consequently, by Lemma 2.1 , its subring $\Theta$ is also not isomorphic to a $2 \times 2$ matrix ring.

In our final example we show that, both in Corollary 3.4(ii) and its more abstract formulation in Theorem 3.3(i), one cannot delete the hypothesis that $\operatorname{det} \alpha=1$.

6.11. ExAmple. Keep the notation of Lemma 6.6. A signed permutation matrix $\bar{\alpha} \in M_{4}(F)$ can be lifted (via $\psi_{2}$ ) to a unit in $M_{2}(\mathbf{H})$ if and only if $\operatorname{det} \bar{\alpha}=1$.

Proof. Combine Lemma 6.6(ii) and Corollary 3.4(ii).

\section{References}

1. H. BAss, Algebraic K-theory (W. A. Benjamin, New York, 1968).

2. A. W. Chatters, 'Representation of tiled matrix rings as full matrix rings', Math. Proc. Cambridge Philos. Soc. 105 (1989) 67-72.

3. A. W. Chatters, 'Matrices, idealisers and integer quaternions', J. Algebra 150 (1992) 45-56.

4. A. W. Chatters, 'Non-isomorphic rings with isomorphic matrix rings', Proc. Edinburgh Math. Soc. 36 (1993) 339-348.

5. S. C. Coutinho, 'Generating modules efficiently over non-commutative Noetherian rings', Trans. Amer. Math. Soc. 323 (1991) 843-856.

6. L. E. DiCKSON, Algebras and their arithmetics (Stechert, New York, 1938).

7. R. M. GURALNiCK and L. S. LEVY, 'Presentations of modules when ideals need not be principal', Illinois J. Math 32 (1988) 593-653.

8. R. M. Guralnick and L. S. Levy, 'Cancellation and direct summands in dimension 1', J. Algebra 142 (1991) 310-347. 
9. M. P. Holland and J. T. STAFFORD, 'Differential operators on rational projective curves', $J$. Algebra 147 (1992) 176-244.

10. N. JaCOBSON, Structure of rings (American Mathematical Society, Providence, R.I., 1956).

11. N. JACOBSON, Basic algebra II (Freeman, San Francisco, 1980).

12. L. S. LEVY, 'Decomposing pairs of modules', Trans. Amer. Math. Soc. 122 (1966) 64-80.

13. L. S. LeVY, 'Direct-sum cancellation and genus class groups', Methods in module theory (eds G. Abrams, J. Haefner, K. Rangaswamy, Dekker, New York, 1992), pp. 213-218.

14. k. S. Levy and J. C. Robson, 'Matrices and pairs of modules', J. Algebra 29 (1974) 427-454.

15. J. C. MCCONNELl and J. C. RoBson, Noncommutative Noetherian rings (Wiley-Interscience, Chichester, 1987).

16. J. C. RoBson, 'Noncommutative Dedekind rings', J. Algebra 9 (1968) 249-265.

17. J. C. Rosson, 'Recognition of matrix rings', Comm. Algebra 19(7) (1991) 2113-2124.

18. J. R. SILVESTER, Introduction to algebraic $K$-theory (Chapman and Hall, London, 1981).

19. J. T. STAFFORD, 'Generating modules efficiently: algebraic K-theory for noncommutative Noetherian rings', J. Algebra 69 (1981) 312-346.

20. J. T. STAFFORd, 'Stably free, projective right ideals', Compositio Math. 54 (1985) 63-78.

21. J. H. M. Wedderburn, 'Non-commutative domains of integrity', J. Reine Angew. Math. 167 (1932) $129-141$.

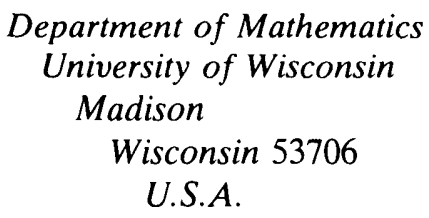

\author{
Department of Pure Mathematics \\ University of Leeds \\ Leeds LS2 $9 \mathrm{JT}$
}

\author{
Department of Mathematics \\ University of Michigan \\ Ann Arbor \\ Michigan 48109 \\ U.S.A.
}

